Geometry $\&$ Topology

Volume 9 (2005) 1253-1293

Published: 24 July 2005

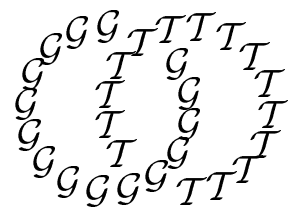

\title{
The colored Jones function is $q$-holonomic
}

\author{
Stavros Garoufalidis \\ Thang T Q LÊ \\ School of Mathematics, Georgia Institute of Technology \\ Atlanta, GA 30332-0160, USA \\ Email: stavros@math.gatech.edu, letu@math.gatech.edu
}

URL: http://www.math.gatech.edu/ stavros

\begin{abstract}
A function of several variables is called holonomic if, roughly speaking, it is determined from finitely many of its values via finitely many linear recursion relations with polynomial coefficients. Zeilberger was the first to notice that the abstract notion of holonomicity can be applied to verify, in a systematic and computerized way, combinatorial identities among special functions. Using a general state sum definition of the colored Jones function of a link in 3-space, we prove from first principles that the colored Jones function is a multisum of a $q$-proper-hypergeometric function, and thus it is $q$-holonomic. We demonstrate our results by computer calculations.
\end{abstract}

\section{AMS Classification numbers Primary: 57N10}

Secondary: 57M25

Keywords: Holonomic functions, Jones polynomial, Knots, WZ algorithm, quantum invariants, $D$-modules, multisums, hypergeometric functions

Proposed: Walter Neumann

Seconded: Joan Birman, Vaughan Jones
Received: 28 October 2004

Revised: 20 July 2005 


\section{Introduction}

\subsection{Zeilberger meets Jones}

The colored Jones function of a framed knot $\mathcal{K}$ in $3-$ space

$$
J_{\mathcal{K}}: \mathbb{N} \longrightarrow \mathbb{Z}\left[q^{ \pm 1 / 4}\right]
$$

is a sequence of Laurent polynomials that essentially measures the Jones polynomial of a knot and its cables. This is a powerful but not well understood invariant of knots. As an example, the colored Jones function of the 0 -framed right-hand trefoil is given by

$$
J_{\mathcal{K}}(n)=\frac{q^{1 / 2-n / 2}}{1-q^{-1}} \sum_{k=0}^{n-1} q^{-k n}\left(1-q^{-n}\right)\left(1-q^{1-n}\right) \ldots\left(1-q^{k-n}\right) .
$$

Here $J_{\mathcal{K}}(n)$ denotes the Jones polynomial of the 0 -framed knot $\mathcal{K}$ colored by the $n$-dimensional irreducible representation of $\mathfrak{s l}_{2}$, and normalized by $J_{\text {unknot }}(n)=\left(q^{n / 2}-q^{-n / 2}\right) /\left(q^{1 / 2}-q^{-1 / 2}\right)$.

Only a handful of knots have such a simple formula. However, as we shall see all knots have a multisum formula. Another way to look at the colored Jones function of the trefoil is via the following 3-term recursion formula:

$$
J_{\mathcal{K}}(n)=\frac{q^{n-1}+q^{4-4 n}-q^{-n}-q^{1-2 n}}{q^{1 / 2}\left(q^{n-1}-q^{2-n}\right)} J_{\mathcal{K}}(n-1)+\frac{q^{4-4 n}-q^{3-2 n}}{q^{2-n}-q^{n-1}} J_{\mathcal{K}}(n-2)
$$

with initial conditions: $J_{\mathcal{K}}(0)=0, \quad J_{\mathcal{K}}(1)=1$.

In this paper we prove that the colored Jones function of any knot satisfies a linear recursion relation, similar to the above one. For a few knots this was obtained by Gelca and his colleagues [13, 14. (In [13] a more complicated 5 -term recursion formula for the trefoil was established).

Discrete functions that satisfy a nontrivial difference recursion relation are known by another name: they are $q$-holonomic.

Holonomic functions were introduced by IN Bernstein [2, 3] and M Saito. The latter coined the term holonomic, that is a function which is entirely determined by the law of its differential equation, together with finitely many initial conditions. Bernstein used holonomic functions to prove a conjecture of Gelfand on the analytic continuation of operators. Holonomicity and the related notion of $D$-modules are a tool in studying linear differential equations from the point 
of view of algebra (differential Galois theory), algebraic geometry, and category theory. For an excellent introduction on holonomic functions and their properties, see [5] and [7].

Our approach to the colored Jones function owes greatly to Zeilberger's work. Zeilberger noticed that the abstract notion of holonomicity can be applied to verify, in a systematic and computerized way, combinatorial identities among special functions, 35] and also 33, 28.

A starting point for Zeilberger, the so-called operator approach, is to replace functions by the recursion relations that they satisfy. This idea leads in a natural way to noncommutative algebras of operators that act on a function, together with left ideals of annihilating operators.

To explain this idea concretely, consider the operators $E$ and $Q$ which act on a discrete function (that is, a function of a discrete variable $n$ ) $f: \mathbb{N} \longrightarrow \mathbb{Z}\left[q^{ \pm}\right]$ by:

$$
(Q f)(n)=q^{n} f(n) \quad(E f)(n)=f(n+1) .
$$

It is easy to see that $E Q=q Q E$, and that $E, Q$ generate a noncommutative $q-$ Weyl algebra generated by noncommutative polynomials in $E$ and $Q$, modulo the relation $E Q=q Q E$ :

$$
\mathcal{A}=\mathbb{Z}\left[q^{ \pm}\right]\langle Q, E\rangle /(E Q=q Q E)
$$

Given a discrete function $f$ as above, consider the recursion ideal $\mathcal{I}_{f}=\{P \in$ $\mathcal{A} \mid P f=0\}$. It is easy to see that it is a left ideal of the $q$-Weyl algebra. We say that $f$ is $q$-holonomic iff $\mathcal{I}_{f} \neq 0$.

In this paper we prove that:

Theorem 1 The colored Jones function of every knot is q-holonomic.

Theorem 1 and its companion Theorem 2 are effective, as their proof reveals.

\section{Theorem 2}

(a) The E-order of the colored Jones function of a knot is bounded above by an exponential function in the number of crossings.

(b) For every knot $\mathcal{K}$ there exist a natural number $n(\mathcal{K})$, such that $n(\mathcal{K})$ initial values of the colored Jones function determine the colored Jones function of $\mathcal{K}$. In other words, the colored Jones function is determined by a finite list. $n(\mathcal{K})$ is bounded above by an exponential function in the number of crossings. 
Computer calculations are given in Section 6. In relation to (b) above, notice that the $q$-Weyl algebra is noetherian; thus every left ideal is finitely generated. The theorem states more, namely that the we can compute (via elimination) a basis for the recursion ideal of the colored Jones function of a knot.

Let us end the introduction with some remarks.

Remark 1.1 The colored Jones function can be defined for every simple Lie algebra $\mathfrak{g}$. Our proof of Theorem 1 generalizes and proves that the $\mathfrak{g}$-colored Jones function of a knot is $q$-holonomic (except for $G_{2}$ ), see Theorem [6 below.

Remark 1.2 The colored Jones function can be defined for colored links in 3-space. Our proof of Theorem 1 proves that the colored Jones function of a link is $q$-holonomic in all variables, see Section 3.1 .

Remark 1.3 It is well known that computing $J(n)$ for any fixed $n>1$ is a $\# P$-complete problem. Theorem 1 claims that this sequence of \# $\mathrm{P}$-complete problems is no worse than any of its terms.

Remark 1.4 The proof of Theorem 1 indicates that many statistical mechanics models, with complicated partition functions that depend on several variables, are holonomic, provided that their local weights are holonomic. This observation may be of interest to statistical mechanics.

\subsection{Synonymous notions to holonomicity}

We have chosen to phrase the results of our paper mostly using the high-school language of linear recursion relations. We could have used synonymous terms such as linear $q$-difference equations, or $q$-holonomic functions, or $D$-modules, or maximally overdetermined systems of linear PDEs which is more common in the area of algebraic analysis, see for example [24]. The geometric notion of $D$ modules gives rise to geometric invariants of knots, such as the characteristic variety introduced by the first author in [11. The characteristic variety is determined by the colored Jones function of a knot and is conjectured to be isomorphic to the $\mathfrak{s l}_{2}(\mathbb{C})$-character variety of a knot, viewed from the boundary torus. This, so-called $A J$ Conjecture, formulated by the first author is known to hold for all torus knots (due to Hikami, 19]), and infinitely many 2-bridge knots (due to the second-author, 21]).

Thus, there is nontrivial geometry encoded in the linear recursion relations of the colored Jones function of a knot. 


\subsection{Plan of the proof}

In Section 2 we discuss in detail the notion of a $q$-holonomic function. We give examples of $q$-holonomic functions (our building blocks), together with rules that create $q$-holonomic functions from known ones.

In Section 3, we discuss the colored Jones function of a link in 3-space, using state sums associated to a planar projection of the link. The colored Jones function is built out of local building blocks (namely, $R$-matrices) associated to the crossings, which are assembled together in a way dictated by the planar projection. The main observation is that the $R$-matrix is $q$-holonomic in all variables, and that the assembly preserves $q$-holonomicity. Theorem 1 follows. As a bonus, we present the colored Jones function as a multisum of a $q$-proper hypergeometric function.

In Section 4 we show that the cyclotomic function of a knot (a reparametrization of the colored Jones function, introduced by Habiro, with good integrality properties) is $q$-holonomic, too. We achieve this by studying explicitly a change of basis for representations of $\mathfrak{s l}_{2}$.

In Section 5 we give a theoretical review about complexity and computability of recursion relations of $q$-holonomic functions, following Zeilberger. These ideas solve the problem of finding recursion relations of $q$-holonomic functions which are given by multisums of $q$ proper hypergeometric functions. It is a fortunate coincidence (?) that the colored Jones function can be presented by such a multisum, thus we can compute its recursion relations. Theorem 2 follows.

Section [6] is a computer implementation of the previous section, where we use Mathematica packages developed by A Riese.

In Section 7 we discuss the $\mathfrak{g}$-colored Jones function of a knot, associated to a simple Lie algebra $\mathfrak{g}$. Our goal is to prove that the $\mathfrak{g}$-colored Jones function is $q$-holonomic in all variables (see Theorem 6). In analogy with the $\mathfrak{g}=\mathfrak{s l}_{2}$ case, we need to show that the local building block, the $R$-matrix, is $q$-holonomic in all variables. This is a trip to the world of quantum groups, which takes up the rest of the section, and ends with an appendix which computes (by brute-force) structure constants of quantized enveloping Lie algebras in the rank 2 case.

\subsection{Acknowledgements}

The authors wish to express their gratitude to D Zeilberger who introduced them to the wonderful world of holonomic functions and its relation to discrete mathematics. Zeilberger is a philosophical co-author of the present paper. 
In addition, the authors wish to thank A Bernstein, G Lusztig, and N Xie for help in understanding quantum group theory, G Masbaum and $\mathrm{K}$ Habiro for help in a formula of the cyclotomic expansion of the colored Jones polynomial, and D Bar-Natan and A Riese for computer implementations.

The authors were supported in part by National Science Foundation.

\section{$2 \quad q$-holonomic and $q$-hypergeometric functions}

Theorem 1 follows from the fact that the colored Jones function can be built from elementary blocks that are $q$-holonomic, and the operations that patch the blocks together to give the colored Jones function preserve $q$-holonomicity.

IN Bernstein defined the notion of holonomic functions $f: \mathbb{R}^{r} \longrightarrow \mathbb{C}$, 2, 3. For an excellent and complete account, see Bjork 4. Zeilberger's brilliant idea was to link the abstract notion of holonomicity to the concrete problem of algorithmically proving combinatorial identities among hypergeometric functions, see [35, 33] and also [28]. This opened an entirely new view on combinatorial identities.

Sabbah extended Bernstein's approach to holonomic functions and defined the notion of a $q$-holonomic function, see [31] and also [6].

\section{$2.1 q$-holonomicity in many variables}

We briefly review here the definition of $q$-holonomicity. First of all, we need an $r$-dimensional version of the $q$-Weyl algebra. Consider the operators $E_{i}$ and $Q_{j}$ for $1 \leq i, j \leq r$ which act on discrete functions $f: \mathbb{N}^{r} \longrightarrow \mathbb{Z}\left[q^{ \pm}\right]$by:

$$
\begin{aligned}
& \left(Q_{i} f\right)\left(n_{1}, \ldots, n_{r}\right)=q^{n_{i}} f\left(n_{1}, \ldots, n_{r}\right) \\
& \left(E_{i} f\right)\left(n_{1}, \ldots, n_{r}\right)=f\left(n_{1}, \ldots, n_{i-1}, n_{i}+1, n_{i+1}, \ldots, n_{r}\right) .
\end{aligned}
$$

It is easy to see that the following relations hold:

$$
\begin{array}{ll}
Q_{i} Q_{j}=Q_{j} Q_{i} & E_{i} E_{j}=E_{j} E_{i} \\
Q_{i} E_{j}=E_{j} Q_{i} \text { for } i \neq j & E_{i} Q_{i}=q Q_{i} E_{i}
\end{array}
$$

We define the $q-$ Weyl algebra $\mathcal{A}_{r}$ to be a noncommutative algebra with presentation

$$
\mathcal{A}_{r}=\frac{\mathbb{Z}\left[q^{ \pm 1}\right]\left\langle Q_{1}, \ldots, Q_{r}, E_{1}, \ldots, E_{r}\right\rangle}{\left(\operatorname{Rel}_{q}\right)}
$$


Given a discrete function $f$ with domain $\mathbb{N}^{r}$ or $\mathbb{Z}^{r}$ and target space a $\mathbb{Z}\left[q^{ \pm 1}\right]$ module, one can define the left ideal $\mathcal{I}_{f}$ in $\mathcal{A}_{r}$ by

$$
\mathcal{I}_{f}:=\left\{P \in \mathcal{A}_{r} \mid P f=0\right\} .
$$

If we want to determine a function $f$ by a finite list of initial conditions, it does not suffice to ensure that $f$ satisfies one nontrivial recursion relation if $r \geq 2$. The key notion that we need instead is $q$-holonomicity.

Intuitively, a discrete function $f: \mathbb{N}^{r} \longrightarrow \mathbb{Z}\left[q^{ \pm}\right]$is $q$-holonomic if it satisfies a maximally overdetermined system of linear difference equations with polynomial coefficients. The exact definition of holonomicity is through homological dimension, as follows.

Suppose $M=\mathcal{A}_{r} / I$, where $I$ is a left $\mathcal{A}_{r}$-module. Let $F_{m}$ be the sub-space of $\mathcal{A}_{r}$ spanned by polynomials in $Q_{i}, E_{i}$ of total degree $\leq m$. Then the module $\mathcal{A}_{r} / I$ can be approximated by the sequence $F_{m} /\left(F_{m} \cap I\right), m=1,2, \ldots$. It turns out that, for $m>>1$, the dimension (over the fractional field $\mathbb{Q}(q)$ ) of $F_{m} /\left(F_{m} \cap I\right)$ is a polynomial in $m$ whose degree $d(M)$ is called the homological dimension of $M$.

Bernstein's famous inequality (proved by Sabbah in the $q$-case, 31]) states that $d(M) \geq r$, if $M \neq 0$ and $M$ has no monomial torsions, ie, any non-trivial element of $M$ cannot be annihilated by a monomial in $Q_{i}, E_{i}$. Note that the left $\mathcal{A}_{r}$-module $M_{f}:=\mathcal{A}_{r} \cdot f \cong \mathcal{A}_{r} / \mathcal{I}_{f}$ does not have monomial torsion.

Definition 2.1 We say that a discrete function $f$ is $q$-holonomic if $d\left(M_{f}\right) \leq r$.

Note that if $d\left(M_{f}\right) \leq r$, then by Bernstein's inequality, either $M_{f}=0$ or $d\left(M_{f}\right)=r$. The former can happen only if $f=0$.

Although we will not use in this paper, let us point out an alternative cohomological definition of dimension for a finitely generated $\mathcal{A}_{r}$ module $M$. Let us define

$$
c(M):=\min \left\{j \in \mathbb{N} \mid \operatorname{Ext}_{\mathcal{A}_{r}}^{j}\left(M, \mathcal{A}_{r}\right) \neq 0\right\} .
$$

Then the homological dimension $d(M):=2 r-c(M)$ equals to the dimension $d(M)$ defined above.

Closely related to $\mathcal{A}_{r}$ is the q-torus algebra $\mathcal{T}_{r}$ with presentation

$$
\mathcal{T}_{r}=\frac{\mathbb{Z}\left[q^{ \pm 1}\right]\left\langle Q_{1}^{ \pm 1}, \ldots, Q_{r}^{ \pm 1}, E_{1}^{ \pm 1}, \ldots, E_{r}^{ \pm 1}\right\rangle}{\left(\operatorname{Rel}_{q}\right)} .
$$

Elements of $\mathcal{T}_{r}$ acts on the set of functions with domain $\mathbb{Z}^{r}$, but not on the set of functions with domain $\mathbb{N}^{r}$. Note that $\mathcal{T}_{r}$ is simple, but $\mathcal{A}_{r}$ is not. If $I$ is a left ideal of $\mathcal{T}_{r}$ then the dimension of $\mathcal{T}_{r} / I$ is equal to that of $\mathcal{A}_{r} /\left(I \cap A_{r}\right)$. 


\subsection{Assembling $q$-holonomic functions}

Despite the unwelcoming definition of $q$-holonomic functions, in this paper we will use not the definition itself, but rather the closure properties of the set of $q$-holonomic functions under some natural operations.

\section{Fact 0}

- Sums and products of $q$-holonomic functions are $q$-holonomic.

- Specializations and extensions of $q$-holonomic functions are $q$-holonomic. In other words, if $f\left(n_{1}, \ldots, n_{m}\right)$ is $q$-holonomic, the so are the functions

$$
\text { and } \quad \begin{aligned}
g\left(n_{2}, \ldots, n_{m}\right) & :=f\left(a, n_{2}, \ldots, n_{m}\right) \\
h\left(n_{1}, \ldots, n_{m}, n_{m+1}\right) & :=f\left(n_{1}, \ldots, n_{m}\right) .
\end{aligned}
$$

- Diagonals of $q$-holonomic functions are $q$-holonomic. In other words, if $f\left(n_{1}, \ldots, n_{m}\right)$ is $q$-holonomic, then so is the function

$$
g\left(n_{2}, \ldots, n_{m}\right):=f\left(n_{2}, n_{2}, n_{3}, \ldots, n_{m}\right) .
$$

- Linear substitution. If $f\left(n_{1}, \ldots, n_{m}\right)$ is $q$-holonomic, then so is the function, $g\left(n_{1}^{\prime}, \ldots, n_{m^{\prime}}^{\prime}\right)$, where each $n_{j}^{\prime}$ is a linear function of $n_{i}$.

- Multisums of $q$-holonomic functions are $q$-holonomic. In other words, if $f\left(n_{1}, \ldots, n_{m}\right)$ is $q$-holonomic, the so are the functions $g$ and $h$, defined by

$$
\begin{aligned}
g\left(a, b, n_{2}, \ldots, n_{m}\right) & :=\sum_{n_{1}=a}^{b} f\left(n_{1}, n_{2}, \ldots, n_{m}\right) \\
h\left(a, n_{2}, \ldots, n_{m}\right) & :=\sum_{n_{1}=a}^{\infty} f\left(n_{1}, n_{2}, \ldots, n_{m}\right)
\end{aligned}
$$

(assuming that the latter sum is finite for each $a$ ).

For a user-friendly explanation of these facts and for many examples, see 35, 33. and $[28$.

\subsection{Examples of $q$-holonomic functions}

Here are a few examples of $q$-holonomic functions. In fact, we will encounter only sums, products, extensions, specializations, diagonals, and multisums of these functions. In what follows we usually extend the ground ring $\mathbb{Z}\left[q^{ \pm 1}\right]$ to 
the fractional field $\mathbb{Q}\left(q^{1 / D}\right)$, where $D$ is a positive integer. We also use $v$ to denote a root of $q, v^{2}=q$.

For $n, k \in \mathbb{Z}$, let

$$
\begin{gathered}
\{n\}:=v^{n}-v^{-n}, \quad[n]:=\frac{\{n\}}{\{1\}}, \quad[n] !:=\prod_{i=1}^{n}[i], \quad\{n\} !:=\prod_{i=1}^{n}\{i\} \\
\{n\}_{k}:= \begin{cases}\prod_{i=1}^{k}\{n-i+1\}, & \text { if } k \geq 0 \\
0 & \text { if } k<0\end{cases} \\
{\left[\begin{array}{l}
n \\
k
\end{array}\right]:=\left\{\begin{array}{ll}
\frac{\{n\}_{k}}{\{k\}_{k}} & \text { if } k \geq 0 \\
0 & \text { if } k<0
\end{array} .\right.}
\end{gathered}
$$

The first four functions are $q$-holonomic in $n$, and the last two, as well as the delta function $\delta_{n, k}$, are $q$-holonomic in both $n$ and $k$.

\section{$2.4 q$-hypergeometric functions}

Definition 2.2 A discrete function $f: \mathbb{Z}^{r} \longrightarrow \mathbb{Q}(q)$ is q-hypergeometric iff $E_{i} f / f \in \mathbb{Q}\left(q, q^{n_{1}}, \ldots, q^{n_{r}}\right)$ for all $i=1, \ldots, r$.

In that case, we know generators for the annihilation ideal of $f$. Namely, let $E_{i} f / f=\left.\left(R_{i} / S_{i}\right)\right|_{Q_{i}=q^{n_{i}}}$ for $R_{i}, S_{i} \in \mathbb{Z}\left[q, Q_{1}, \ldots, Q_{r}\right]$. Then, the annihilation ideal of $f$ is generated by $S_{i} E_{i}-R_{i}$.

All the functions in the previous subsections are $q$-hypergeometric.

Unfortunately, $q$-hypergeometric functions are not always $q$-holonomic. For example, $(n, k) \longrightarrow 1 /\left[n^{2}+k^{2}\right]$ ! is $q$-hypergeometric but not $q$-holonomic. However, $q$-proper-hypergeometric functions are $q$-holonomic. The latter were defined by Wilf-Zeilberger as follows, 33, Sec.3.1]:

Definition 2.3 A proper q-hypergeometric discrete function is one of the form

$$
F(n, \mathbf{k})=\frac{\prod_{s}\left(A_{s} ; q\right)_{a_{s} n+\mathbf{b}_{s} \cdot \mathbf{k}+c_{s}}}{\prod_{t}\left(B_{t} ; q\right)_{u_{t} n+\mathbf{v}_{t} \cdot \mathbf{k}+w_{t}}} q^{A(n, \mathbf{k})} \xi^{\mathbf{k}}
$$

where $A_{s}, B_{t} \in \mathbb{K}=\mathbb{Q}(q), a_{s}, u_{t}$ are integers, $\mathbf{b}_{s}, \mathbf{k}_{s}$ are vectors of $r$ integers, $A(n, \mathbf{k})$ is a quadratic form, $c_{s}, w_{s}$ are variables and $\xi$ is an $r$ vector of elements in $\mathbb{K}$. Here, as usual

$$
(A ; q)_{n}:=\prod_{i=0}^{n-1}\left(1-A q^{i}\right) .
$$




\section{The colored Jones function for $\mathfrak{s l}_{2}$}

\subsection{Proof of Theorem 1 for links}

We will formulate and prove an analog of Theorem 1 (see Theorem 3 below) for colored links. Our proof will use a state-sum definition of the colored Jones function, coming from a representation of the quantum group $U_{q}\left(\mathfrak{s l}_{2}\right)$, as was discovered by Reshetikhin and Turaev in [29, 32].

Suppose $L$ is a framed, oriented link of $p$ components. Then the colored Jones function $J_{L}: \mathbb{N}^{p} \rightarrow \mathbb{Z}\left[q^{ \pm 1 / 4}\right]=\mathbb{Z}\left[v^{ \pm 1 / 2}\right]$ can be defined using the representations of braid groups coming from the quantum group $U_{q}\left(\mathfrak{s l}_{2}\right)$.

Theorem 3 The colored Jones function $J_{L}$ is $q$-holonomic.

Proof We will present the definition of $J_{L}$ in the form most suitable for us. Let $V(n)$ be the $n$-dimensional vector space over the field $\mathbb{Q}\left(v^{1 / 2}\right)$ with basis $\left\{e_{0}, e_{1}, \ldots, e_{n-1}\right\}$, with $V(0)$ the zero vector space.

Fix a positive integer $m$. A linear operator

$$
A: V\left(n_{1}\right) \otimes \cdots \otimes V\left(n_{m}\right) \rightarrow V\left(n_{1}^{\prime}\right) \otimes \cdots \otimes V\left(n_{m}^{\prime}\right)
$$

can be described by the collection

$$
A_{a_{1}, \ldots, a_{m}}^{b_{1}, \ldots, b_{m}} \in \mathbb{Q}\left(v^{1 / 2}\right)
$$

where

$$
A\left(e_{a_{1}} \otimes \cdots \otimes e_{a_{m}}\right)=\sum_{b_{1}<n_{1}^{\prime}, \ldots, b_{m}<n_{m}^{\prime}} A_{a_{1}, \ldots, a_{m}}^{b_{1}, \ldots, b_{m}} e_{b_{1}} \otimes \cdots \otimes e_{j_{m}}
$$

We will call $\left(a_{1}, \ldots, a_{m}, b_{1}, \ldots, b_{m}\right)$ the coordinates of the matrix entry $A_{a_{1}, \ldots, a_{m}}^{b_{1}, \ldots, b_{m}}$ of $A$, with respect to the given basis.

The building block of our construction is a pair of functions $f_{ \pm}: \mathbb{Z}^{5} \rightarrow \mathbb{Z}\left[v^{ \pm 1 / 2}\right]$, given by

$$
\begin{aligned}
& f_{+}\left(n_{1}, n_{2} ; a, b, k\right) \\
& \quad:=(-1)^{k} v^{-\left(\left(n_{1}-1-2 a\right)\left(n_{2}-1-2 b\right)+k(k-1)\right) / 2}\left[\begin{array}{c}
b+k \\
k
\end{array}\right]\left\{n_{1}-1+k-a\right\}_{k}, \\
& f_{-}\left(n_{1}, n_{2} ; a, b, k\right) \\
& \quad:=v^{\left(\left(n_{1}-1-2 a-2 k\right)\left(n_{2}-1-2 b+2 k\right)+k(k-1)\right) / 2}\left[\begin{array}{c}
a+k \\
k
\end{array}\right]\left\{n_{2}-1+k-b\right\}_{k} .
\end{aligned}
$$


The reader should not focus on the actual, cumbersome formulas. The main point is that:

\section{Fact 1}

- $f_{+}$and $f_{-}$are $q$-proper hypergeometric and thus $q$-holonomic in all variables.

For each pair $\left(n_{1}, n_{2}\right) \in \mathbb{N}^{2}$ we define two operators

$$
\mathcal{B}_{+}\left(n_{1}, n_{2}\right), \mathcal{B}_{-}\left(n_{1}, n_{2}\right): V\left(n_{1}\right) \otimes V\left(n_{2}\right) \rightarrow V\left(n_{2}\right) \otimes V\left(n_{1}\right)
$$

by

$$
\begin{aligned}
\left(\mathcal{B}_{+}\left(n_{1}, n_{2}\right)\right)_{a, b}^{c, d} & :=f_{+}\left(n_{1}, n_{2} ; a, b, c-b\right) \delta_{c-b, a-d}, \\
\left(\mathcal{B}_{-}\left(n_{1}, n_{2}\right)\right)_{a, b}^{c, d} & :=f_{+}\left(n_{1}, n_{2} ; a, b, b-c\right) \delta_{c-b, a-d},
\end{aligned}
$$

where $\delta_{x, y}$ is Kronecker's delta function. Although the coordinates $(a, b, c, d)$ of the entry $\left.\mathcal{B}_{ \pm}\left(n_{1}, n_{2}\right)\right)_{a, b}^{c, d}$ of the operators $\mathcal{B}_{ \pm}\left(n_{1}, n_{2}\right)$ are defined for $0 \leq$ $a, b \leq n_{1}$ and $0 \leq c, d \leq n_{2}$, the above formula makes sense for all non-negative integers $a, b, c, d$. This will be important for us. The following lemma is obvious.

Lemma 3.1 The discrete functions $\mathcal{B}_{ \pm}\left(n_{1}, n_{2}\right)_{a, b}^{c, d}$ are $q$-holonomic with respect to the variables $\left(n_{1}, n_{2}, a, b, c, d\right)$.

If we identify $V(n)$ with the simple $n$-dimensional $U_{q}\left(\mathfrak{s l}_{2}\right)$-module, with $e_{i}, i=$ $0, \ldots, n-1$ being the standard basis, then $\mathcal{B}_{+}\left(n_{1}, n_{2}\right), \mathcal{B}_{-}\left(n_{1}, n_{2}\right)$ are respectively the braiding operator and its inverse acting on $V\left(n_{1}\right) \otimes V\left(n_{2}\right)$. This fact follows from the formula of the $R$-matrix, say, in [17, Chapter 3]. In particular, $\mathcal{B}_{-}\left(n_{1}, n_{2}\right)$ is the inverse of $\mathcal{B}_{+}\left(n_{1}, n_{2}\right)$. If one allows $a, b, c, d$ in $\mathcal{B}_{ \pm}\left(n_{1}, n_{2}\right)_{a, b}^{c, d}$ to run the set $\mathbb{N}$, then $\mathcal{B}_{ \pm}\left(n_{1}, n_{2}\right)_{a, b}^{c, d}$ define the braid action on the Verma module corresponding to $V\left(n_{1}\right), V\left(n_{2}\right)$.

Let $B_{m}$ be the braid group on $m$ strands, with standard generators $\sigma_{1}, \ldots, \sigma_{m-1}$ :

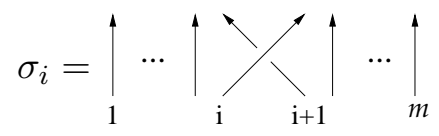

For each braid $\beta \in B_{m}$ and $\left(n_{1}, \ldots, n_{m}\right) \in \mathbb{N}^{m}$, we will define an operator $\tau(\beta)=\tau(\beta)\left(n_{1}, \ldots, n_{m}\right)$,

$$
\tau(\beta): V\left(n_{1}\right) \otimes \cdots \otimes V\left(n_{m}\right) \rightarrow V\left(n_{\bar{\beta}(1)}\right) \otimes \cdots \otimes V\left(n_{\bar{\beta}(m)}\right),
$$


where $\bar{\beta}$ is the permutation of $\{1, \ldots, m\}$ corresponding to $\beta$. The operator $\tau(\beta)$ is uniquely determined by the following properties: For an elementary braid $\sigma_{i}$, we have:

$$
\tau\left(\sigma_{i}^{ \pm 1}\right)=\mathrm{id}^{\otimes i-1} \otimes \mathcal{B}_{ \pm}\left(n_{i}, n_{i+1}\right) \mathrm{id}^{\otimes m-i-1} .
$$

In addition, if $\beta=\beta^{\prime} \beta^{\prime \prime}$, then $\tau(\beta):=\tau\left(\beta^{\prime}\right) \tau\left(\beta^{\prime \prime}\right)$. It is well-known that $\tau(\beta)$ is well-defined.

From Fact 0 and Lemma 3.1 it follows that

Lemma 3.2 For any braid $\beta \in B_{m}$, the discrete function $\tau(\beta)\left(n_{1}, \ldots, n_{m}\right)$, considered as a function with variables $n_{1}, \ldots, n_{m}$ and all the coordinates of the matrix entry, is q-holonomic.

Let $K$ be the linear endomorphism of $V\left(n_{1}\right) \otimes \cdots \otimes V\left(n_{m}\right)$ defined by

$$
K\left(e_{i_{1}} \otimes \cdots \otimes e_{i_{m}}\right)=v^{n_{1}+\cdots+n_{m}-2 i_{1}-\cdots-2 i_{m}-m} e_{i_{1}} \otimes \cdots \otimes e_{i_{m}} .
$$

The inverse operator $K^{-1}$ is well-defined.

Corollary 3.3 For any braid $\beta \in B_{m}$, the discrete function

$$
\tilde{\tau}(\beta):=\tau(\beta)\left(n_{1}, \ldots, n_{m}\right) \times K^{-1}
$$

is $q$-holonomic in $n_{1}, \ldots, n_{m}$ all all of the coordinates of the matrix entry.

In general, the trace of $\tilde{\tau}(\beta)$ is called the quantum trace of $\tau(\beta)$. Although the target space and source space maybe different, let us define the quantum trace of $\left.\tau(\beta)\left(n_{1}, \ldots, n_{m}\right)\right)$ by

$$
\operatorname{tr}_{q}(\beta)\left(n_{1}, \ldots, n_{m}\right):=\sum_{1 \leq i \leq m} \sum_{0 \leq a_{i}<n_{i}} \tilde{\tau}(\beta)\left(n_{1}, \ldots, n_{m}\right)_{a_{1}, \ldots, a_{m}}^{a_{1}, \ldots, a_{m}} .
$$

It follows from Fact 0 that $\operatorname{tr}_{q}(\beta)\left(n_{1}, \ldots, n_{m}\right)$ is $q$-holonomic in $n_{1}, \ldots, n_{m}$. Restricting this function on the diagonal defined by $n_{i}=n_{\bar{\beta} i}, i=1, \ldots, m$, we get a new function $J_{\beta}$ of $p$ variables, where $p$ is the number of cycles of the permutation $\bar{\beta}$.

Suppose a framed link $L$ can be obtained by closing the braid $\beta$. Then the colored Jones polynomial $J_{L}$ is exactly $J_{\beta}$. Hence Theorem 1 follows.

Remark 3.4 In general, $J_{\mathcal{K}}(n)$ contains the fractional power $q^{1 / 4}$. If $K$ has framing 0 , then $J_{\mathcal{K}^{\prime}}(n):=J_{\mathcal{K}}(n) /[n] \in \mathbb{Z}\left[q^{ \pm 1}\right]$. See $[20$. 
Remark 3.5 There is a variant of the colored Jones function $J_{L^{\prime}}$ of a colored link $L^{\prime}$ where one of the components is broken. If $\beta$ is a braid as above, let us define the broken quantum trace $\operatorname{tr}_{\beta}^{\prime}$ by

$$
\operatorname{tr}_{q}^{\prime}(\beta)\left(n_{1}, \ldots, n_{m}\right):=\left.\sum_{2 \leq i \leq m} \sum_{0 \leq a_{i}<n_{i}} \tilde{\tau}(\beta)\left(n_{1}, \ldots, n_{m}\right)_{a_{1}, \ldots, a_{m}}^{a_{1}, \ldots, a_{m}}\right|_{a_{1}=0} .
$$

Restricting this function on the diagonal defined by $n_{i}=n_{\bar{\beta} i}, i=1, \ldots, m$, we get a new function $J_{\beta^{\prime}}$ of $p$ variables, where $p$ is the number of cycles of the permutation $\bar{\beta}$.

If $L^{\prime}$ denotes the broken link which is the closure of all but the first strand of $\beta$, then the colored Jones function $J_{L^{\prime}}$ of $L^{\prime}$ satisfies $J_{L^{\prime}}=J_{\beta^{\prime}}$.

If $L$ denotes the closure of the broken link $L^{\prime}$, then we have:

$$
J_{L}=J_{L^{\prime}} \times[\lambda]
$$

where $\lambda$ is the color of the broken component of $L^{\prime}$.

\subsection{A multisum formula for the colored Jones function of a knot}

In this section we will give explicit multisum formulas for the $\mathfrak{s l}_{2}$-colored Jones function of a knot. The calculation here is computerized in Section 6 .

Consider a word $w=\sigma_{i_{1}}^{\epsilon_{1}} \ldots \sigma_{i_{c}}^{\epsilon_{c}}$ of length $m$ written in the standard generators $\sigma_{1}, \ldots, \sigma_{s-1}$ of the braid group $B_{m}$ with $m$ strands, where $\epsilon_{i}= \pm 1$ for all $i$.

$w$ gives rise to a braid $\beta \in B_{m}$, and we assume that the closure of $\beta$ is a knot $\mathcal{K}$. Let $\mathcal{K}^{\prime}$ denote long knot which is the closure of all but the first strand of $\beta$.

A coloring of $\mathcal{K}^{\prime}$ is a tuple $\mathbf{k}=\left(k_{1}, \ldots, k_{c}\right)$ of angle variables placed at the crossings of $\mathcal{K}^{\prime}$.

Lemma 3.6 There is a unique way to extend a coloring $\mathbf{k}$ of $\mathcal{K}^{\prime}$ to a coloring of the crossings and part-arcs of $\mathcal{K}^{\prime}$ such that

- around each crossing the following consistency relations are satisfied:
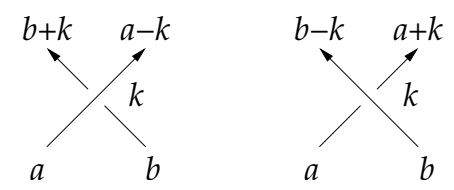

- The color of the lower-left incoming part-arc is 0 . 
Moreover, the labels of the part-arcs are linear forms on $\mathbf{k}$.

Proof Start walking along the long knot starting at the incoming part-arc. At the first crossing, whether over or under, the label of the outgoing part-arc is determined by the label of the ending part-arc and the angle variable of the crossing. Thus, we know the label of the outgoing part-arc of the first crossing. Keep going. Since $\mathcal{K}^{\prime}$ is topologically an interval, the result follows.

For an example, see Figure 1

$$
w=\sigma_{1}^{3}
$$
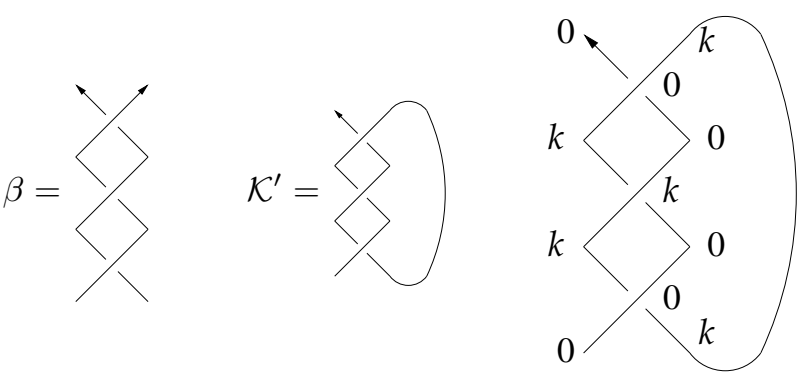

Figure 1: A word $w$, the corresponding braid $\beta$, its long closure $\mathcal{K}^{\prime}$, and a coloring of $\mathcal{K}^{\prime}$

Fix a coloring of $\mathcal{K}^{\prime}$ determined by a vector $\mathbf{k}$. Let $b_{i}(\mathbf{k})$ for $i=1, \ldots, m$ denote the labels of the top part-arcs of $\beta$. Let $x_{j}(\mathbf{k})$ and $y_{j}(\mathbf{k})$ denote the labeling of the left and right incoming part-arcs at the $i$ th crossing of $\mathcal{K}^{\prime}$ for $j=1, \ldots, c$. According to Lemma 3.6. $b_{i}(\mathbf{k}), x_{j}(\mathbf{k})$ and $y_{j}(\mathbf{k})$ are linear forms on $\mathbf{k}$.

It is easy to see that

$$
\operatorname{tr}_{q}^{\prime}(\beta)=\sum_{\mathbf{k} \geq \mathbf{0}} F_{w}(n, \mathbf{k})
$$

where

$$
F_{w}(n, \mathbf{k}):=\prod_{i=2}^{m} v^{\frac{n}{2}-b_{i}(\mathbf{k})} \prod_{j=1}^{c} f_{\operatorname{sgn}\left(\epsilon_{i}\right)}\left(n, n ; x_{j}(\mathbf{k}), y_{j}(\mathbf{k})\right) .
$$

is a $q$-proper hypergeometric function. Remark 3.5 then implies that

Proposition 3.7 The colored Jones function of a long knot $\mathcal{K}^{\prime}$ is a multisum of a $q$-proper hypergeometric function:

$$
J_{\mathcal{K}^{\prime}}(n)=\sum_{\mathbf{k} \geq \mathbf{0}} F_{w}(n, \mathbf{k}) .
$$


Remark 3.8 If a long knot $\mathcal{K}^{\prime}$ is presented by a planar projection $D$ with $c$ crossings (which is not necessarily the closure of a braid), then similar to the above there is a $q$-proper hypergeometric function $F_{D}(n, \mathbf{k})$ of $c+1$ variables such that $J_{\mathcal{K}^{\prime}}(n)=\sum_{\mathbf{k} \geq \mathbf{0}} F_{D}(n, \mathbf{k})$. Of course, $F_{D}$ depends on the planar projection. Occasionally, some of the summation variables can be ignored. This is the case for the right hand-trefoil (where the multisum reduces to a single sum) and the figure eight (where it reduces to a double sum).

D Bar-Natan has kindly provided us with a computerized version of Proposition 3.7. [1].

\section{The cyclotomic function of a knot is $q$-holonomic}

Habiro [15] proved that the colored Jones polynomial (of $\mathfrak{s l}_{2}$ ) can be rearranged in the following convenient form, known as the cyclotomic expansion of the colored Jones polynomial: For every 0 -framed knot $\mathcal{K}$, there exists a function

$$
C_{\mathcal{K}}: \mathbb{Z}_{>0} \rightarrow \mathbb{Z}\left[q^{ \pm 1}\right]
$$

such that

$$
J_{\mathcal{K}}(n)=\sum_{k=1}^{\infty} C_{\mathcal{K}}(k) S(n, k),
$$

where

$$
S(n, k):=\{n+k-1\}_{2 k-1} /\left(v-v^{-1}\right)=\frac{\prod_{n-k+1}^{n+k-1}\left(v^{i}-v^{-i}\right)}{v-v^{-1}} .
$$

Note that $S(n, k)$ does not depend on the knot $\mathcal{K}$. Note that $J$ is determined from $C$ and vice-versa by an upper diagonal matrix, thus $C$ takes values in $\mathbb{Q}(q)$. The difficult part of Habiro's result is $C_{\mathcal{K}}$ takes values in $\mathbb{Z}\left[q^{ \pm}\right]$. The integrality of the cyclotomic function is a crucial ingredient in the study of integrality properties of 3-manifold invariants, [15.

Theorem 4 The cyclotomic function $C_{\mathcal{K}}: \mathbb{N} \rightarrow \mathbb{Z}\left[q^{ \pm}\right]$of every knot $\mathcal{K}$ is q-holonomic.

Proof Habiro showed that $C_{\mathcal{K}}(n)$ is the quantum invariant of the knot $\mathcal{K}$ with color

$$
P^{\prime \prime}(n):=\frac{\prod_{i=1}^{n-1}\left(V(2)-v^{2 i-1}-v^{1-2 i}\right)}{\{2 n-1\}_{2 n-2}},
$$


where $V(n)$ is the unique $n$-dimensional simple $\mathfrak{s l}_{2}$-module, and (retaining Habiro's notation with a shift $n \rightarrow n-1) P^{\prime \prime}(n)$ is considered as an element of the ring of $\mathfrak{s l}_{2}$-modules over $\mathbb{Q}(v)$.

Using induction one can easily prove that

$$
P^{\prime \prime}(n)=\sum_{k=1}^{n} R(n, k) V(k),
$$

where $R(k, n)$ is given by

$$
R(n, k)=(-1)^{n-k} \frac{\{2 k\}}{\{2 n-1\} ![2 n]}\left[\begin{array}{c}
2 n \\
n-k
\end{array}\right] .
$$

We learned this formula from Habiro [15] and Masbaum [25]. Since

$$
C_{\mathcal{K}}(n)=\sum_{k} R(n, k) J_{\mathcal{K}}(k)
$$

and $R(n, k)$ is $q$-proper hypergeometric and thus $q$-holonomic in both variables $n$ and $k$, it follows that $C_{\mathcal{K}}$ is $q$-holonomic.

\section{Complexity}

In this section we show that Theorem 1 is effective. In other words, we give a priori bounds and computations that appear in Theorem 2.

\subsection{Finding a recursion relation for multisums}

Our starting point are multisums of $q$-proper hypergeometric functions. Recall

the definition 2.3 of a $q$-proper hypergeometric function $F(n, \mathbf{k})$ from Section 2.4. and let $G$ denote

$$
G(n):=\sum_{\mathbf{k} \geq \mathbf{0}} F(n, \mathbf{k})
$$

throughout this section.

With the notation of Equation (11), Wilf-Zeilberger show that:

Theorem 5 ([33, Sec.5.2])

(a) $F(n, \mathbf{k})$ satisfies a $k$-free recurrence relation of order at most

$$
J^{\star}:=\frac{\left(4 S T B^{2}\right)^{r}}{r !}
$$


where $B=\max _{s, t}\left\{\left|\mathbf{b}_{s}\right|,\left|\mathbf{v}_{t}\right|,\left|a_{s}\right|,\left|u_{t}\right|\right\}+\max _{\mu, \nu}\left|a_{\mu, \nu}\right|$ where $a_{\mu, \nu}$ are the coefficients of the quadratic form $A$.

(b) Moreover, $G(n)$ satisfies an inhomogeneous recursion relation of order at most $J^{\star}$.

Let us briefly comment on the proof of this theorem. A certificate is an operator of the form

$$
P(E, Q)+\sum_{i=1}^{r}\left(E_{i}-1\right) R_{i}\left(E, E_{1}, \ldots, E_{r}, Q, Q_{1}, \ldots, Q_{r}\right)
$$

that annihilates $F(n, \mathbf{k})$, where $P$ and $R_{i}$ are operators with $P$ a polynomial in $E, Q$, with $P \neq 0$. Here $E$ is the shift operator on $n, E_{i}$ (for $\left.i=1, \ldots, r\right)$ are shift operators in $k_{i}$, and $Q$ is the multiplication operator by $q^{k}$ and $Q_{i}$ (for $i=1, \ldots, r)$ are the multiplication operator by $q^{k_{i}}$, where $\mathbf{k}=\left(k_{1}, \ldots, k_{r}\right)$.

The important thing is that $P(E, Q)$ is an operator that does not depend on the summation variables $\mathbf{k}$. A certificate implies that for all $(n, \mathbf{k})$ we have:

$$
\begin{array}{r}
P(E, Q) F(n, \mathbf{k})+\sum_{i=1}^{r}\left(G_{i}\left(n, k_{1}, \ldots, k_{i-1}, k_{i}+1, k_{i+1}, \ldots, k_{r}\right)-\right. \\
\left.G_{i}\left(n, k_{1}, \ldots, k_{i-1}, k_{i}, k_{i+1}, \ldots, k_{r}\right)\right)=0,
\end{array}
$$

where $G_{i}(n, \mathbf{k})=R_{i} F(n, \mathbf{k})$. Summing over $\mathbf{k} \geq \mathbf{0}$, it follows that $G(n)$ satisfies an inhomogeneous recursion relation $P G=\operatorname{error}(n)$. Here error $(n)$ is a sum of multisums of $q$-proper hypergeometric functions of one variables less. Iterating the process, we finally arrive at a homogeneous recursion relation for $G$.

How can one find a certificate given $F(n, \mathbf{k})$ ? Suppose that $F$ satisfies a $\mathbf{k}-$ free recursion relation $A F=0$, where $A=A\left(E, Q, E_{1}, \ldots, E_{r}\right)$ is an operator that does not depend on the $Q_{i}$. Then, evaluating $A$ at $E_{1}=\ldots E_{r}=1$, we obtain that

$$
A=A(E, Q, 1, \ldots, 1)+\sum_{i=1}^{r}\left(E_{i}-1\right) R_{i}\left(E, Q, E_{1}, \ldots, E_{r}\right)
$$

is a certificate.

How can we find a $\mathbf{k}$-free recursion relation for $F$ ? Let us write

$$
A=\sum_{(i, \boldsymbol{j}) \in S} \sigma_{i, \boldsymbol{j}}(Q) E^{i} E^{\boldsymbol{j}}
$$

where $S$ is a finite set, $\boldsymbol{j}=\left(j_{1}, \ldots, j_{r}\right), E^{\boldsymbol{j}}=E_{1}^{j_{1}} \ldots E_{r}^{j_{r}}$, and $\sigma_{i, \boldsymbol{j}}(Q)$ are polynomial functions in $Q$ with coefficients in $\mathbb{Q}(q)$; see [30]. The condition 
$A F=0$ is equivalent to the equation $(A F) / F=0$. Since $F$ is $q$-proper hypergeometric, the latter equation is the vanishing of a rational function in $Q_{1}, \ldots, Q_{r}$. By cleaning out denominators, this is equivalent to a system of linear equations (namely, the coefficients of monomials in $Q_{i}$ are zero), with unknowns the polynomial functions $\sigma_{i, \boldsymbol{j}}$. For a careful discussion, see [30]. As long as there are more unknowns than equations, the system is guaranteed to have a solution. 33] estimate the number of equations and unknowns in terms of $F(n, \mathbf{k})$, and prove Theorem 5 .

Wilf-Zeilberger programmed the above proof, see [28]. As time passes the algorithms get faster and more refined. For the state-of-the-art algorithms and implementations, see [26, 27] and 30, which we will use below.

Alternative algorithms of noncommutative elimination, using noncommutative Gröbner basis, have been developed by Chyzak and Salvy, [8]. In order for have Gröbner basis, one needs to use the following localization of the $q$-Weyl algebra

$$
\mathcal{B}_{r}=\frac{Q\left(q, Q_{1}, \ldots, Q_{r}\right)\left\langle E_{1}, \ldots, E_{r}\right\rangle}{\left(\operatorname{Rel}_{q}\right)} .
$$

and Gröbner basis [8].

In case $r=1, \mathcal{B}_{1}$ is a principal ideal domain [7, Chapter 2, Exercise 4.5]. In that case one can associate an operator in $\mathcal{B}_{1}$ (unique up to units) that generates that annihilating ideal of $G(n)$. For a conjectural relation between this operator for the $\mathfrak{s l}_{2}$-colored Jones function of a knot and hyperbolic geometry, see [11.

Let us point out however that none of the above algorithms can find generators for the annihilating ideal of the multisum $G(n)$. In fact, it is an open problem how to find generators for the annihilating ideal of $G(n)$ in terms of generators for the annihilating ideal of $F(n, \mathbf{k})$, in theory or in practice. We thank M Kashiwara for pointing this out to us.

\subsection{Upper bounds for initial conditions}

In another direction, one may ask the following question: if a $q$-holonomic function satisfies a nontrivial recursion relation, it follows that it is uniquely determined by a finite number of initial conditions. How many? This was answered by Yen, 34]. If $G$ is a discrete function which satisfies a recursion relation of order $J^{\star}$, consider its principal symbol $\sigma(q, Q)$, that is the coefficient of the leading $E$-term. The principal symbol lies in the commutative ring $\mathbb{Z}\left[q^{ \pm}, Q^{ \pm}\right]$of Laurrent polynomials in two variables $q$ and $Q$. For every $n$, consider the Laurrent polynomial $\sigma\left(q, q^{n}\right) \in \mathbb{Z}\left[q^{ \pm}\right]$. If $\sigma\left(q, q^{n}\right) \neq 0$ for all $n$, 
then $G$ is determined by $J^{\star}$ many initial values. Since $\sigma(q, Q) \neq 0$, it follows that $\sigma\left(q, q^{n}\right) \neq 0$ for large enough $n$. In fact, in [34. Prop.3.1] Yen proves that $\sigma\left(q, q^{n}\right) \neq 0$ if $n>\operatorname{deg}_{q}(\sigma)$, then $\sigma\left(q, q^{n}\right) \neq 0$, where the degree of a Laurrent polynomial in $q$ is the difference between the largest and smallest exponent. Thus, $G$ is determined by $J^{\star \star}:=J^{\star}+\operatorname{deg}_{q}(\sigma)$ initial conditions.

Yen further gives upper bounds for $\operatorname{deg}_{q}(\sigma)$ in terms of the $q$-hypergeometric summand, see [34, Thm.2.9] for single sums. An extension of Yen's work to multisums, gives a priori upper bounds $J^{\star \star}$ in terms of the $q$-hypergeometric summand. These exponential bounds are of theoretical interest only, and in practice much smaller bounds are found by computer.

\subsection{Proof of Theorem 2}

Theorem 2 follows from Proposition 3.7 together with the discussion of Sections 5.1 and 5.2

Our luck with the colored Jones function is that we can identify it with a multisum of a $q$-proper hypergeometric function. Are we really lucky, or is there some deeper explanation? We believe that there is a underlying geometric reason for coincidence, which in a sense explains the underlying geometry of topological quantum field theory. We will postpone to a later publication applications of this principle to Hyperbolic Geometry; 11.

\section{In computer talk}

In this section we will show that Proposition 3.7 can be implemented by computer.

For every knot, one can write down a multisum formula for the colored Jones function, where the summand is $q$-hypergeometric. Occasionally, this multisum formula can be written as a single sum. There are various programs that can compute the recursion relations and their orders for multisums. In maple, one may use qEKHAD developed by Zeilberger 28. In Mathematica, one may use the qZeil.m and qMultiSum.m packages of RISC developed by Paule and Riese [26, 27, 30]. 


\subsection{Recursion relations for the cyclotomic function of twist knots}

The twist knots $K p$ for integer $p$ are shown in Figure 2. Their planar projections have $2|p|+2$ crossings, $2|p|$ of which come from the full twists, and 2 come from the negative clasp.

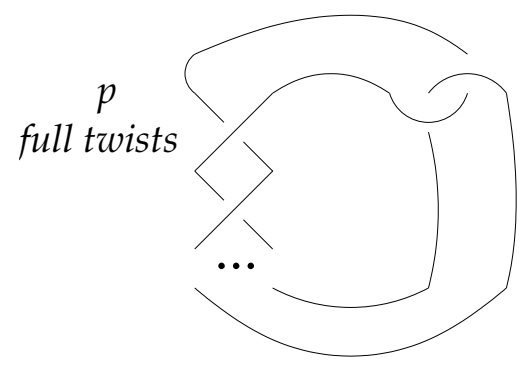

Figure 2: The twist knot $K_{p}$, for integers $p$. For $p=-1$, it is the Figure 8 , for $p=0$ it is the unknot, for $p=1$ it is the left trefoil and for $p=2$ it is the Stevedore's ribbon knot.

Masbaum, 25, following Habiro and Le gives the following formula for the cyclotomic function of a twist knot. Let $c(p, \cdot)$ denote the cyclotomic function of the twist knot $K p$. Rearranging a bit Masbaum's formula [25, Eqn.(35)], we obtain that:

$$
\begin{aligned}
c(p, n) & =(-1)^{n+1} q^{n(n+3) / 2} \\
& \sum_{k=0}^{\infty}(-1)^{k} q^{k(k+1) p+k(k-1) / 2}\left(q^{2 k+1}-1\right) \frac{(q ; q)_{n}}{(q ; q)_{n+k+1}(q ; q)_{n-k}}
\end{aligned}
$$

The above sum has compact support for each $n$. Now, in computer talk, we have:

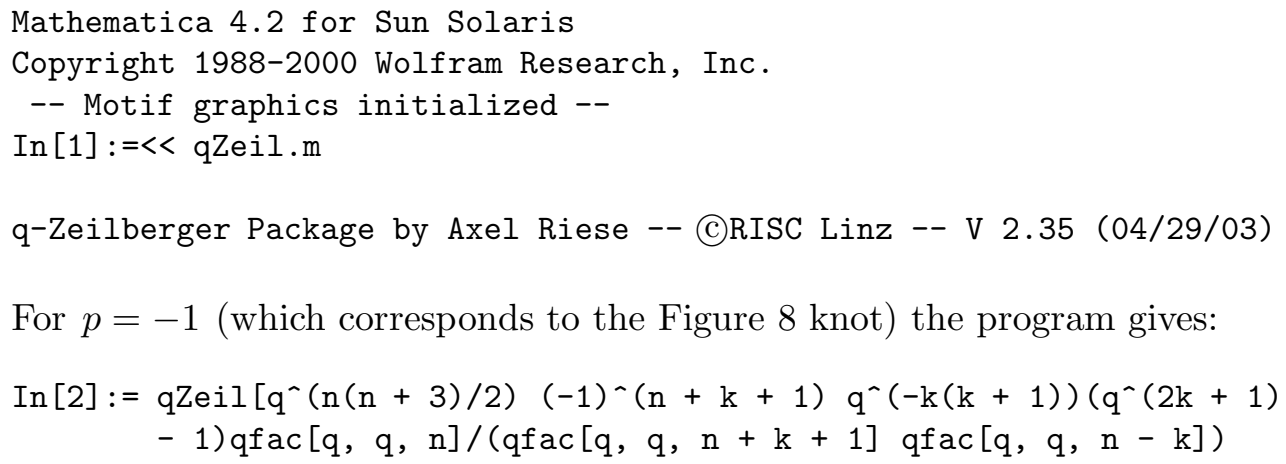

For $p=-1$ (which corresponds to the Figure 8 knot) the program gives: 
$\mathrm{q}^{\wedge}(\mathrm{k}(\mathrm{k}-1) / 2),\{\mathrm{k}, 0$, Infinity $\left.\}, \mathrm{n}, 1\right]$

Out $[2]=\operatorname{SUM}[\mathrm{n}]==\operatorname{SUM}[-1+\mathrm{n}]$

which means that $c(-1, n)=c(-1, n-1)$ in accordance to the discussion after [25. Thm.5.1] which states $c(-1, n)=1$ for all $n$.

For $p=1$ (which corresponds to the left hand trefoil) the program gives:

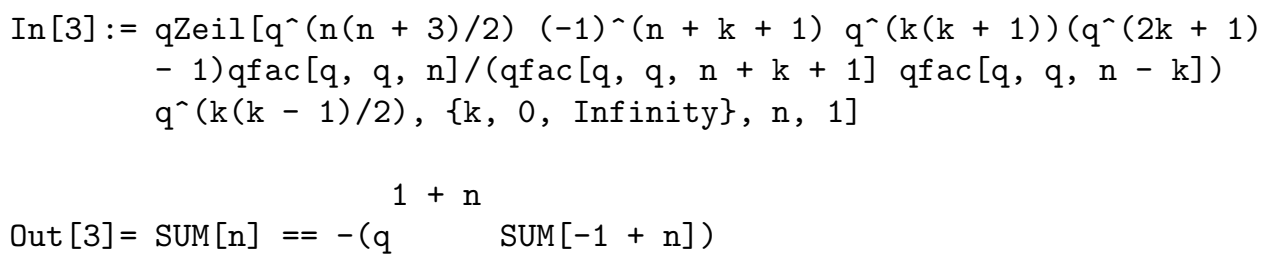

which means that $c(1, n)=-q^{n+1} c(1, n-1)$ in accordance to the discussion after [25, Thm.5.1] which states $c(1, n)=(-1)^{n} q^{n(n+3) / 2}$ for all $n$.

Similarly, for $p=2$ (which corresponds to Stevedore's ribbon knot) the program gives:

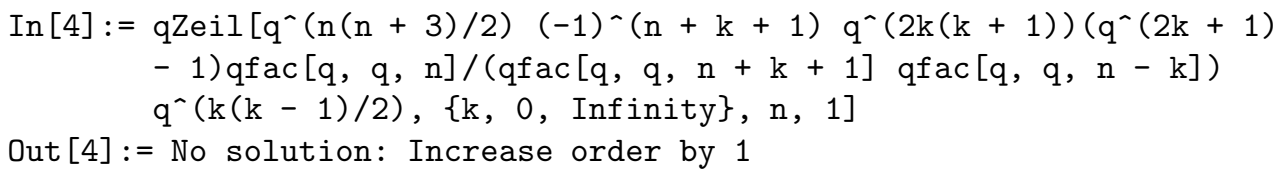

which proves that $c(2, n)$ satisfies no first order recursion relation. It does satisfy a second order recursion relation, as we find by:

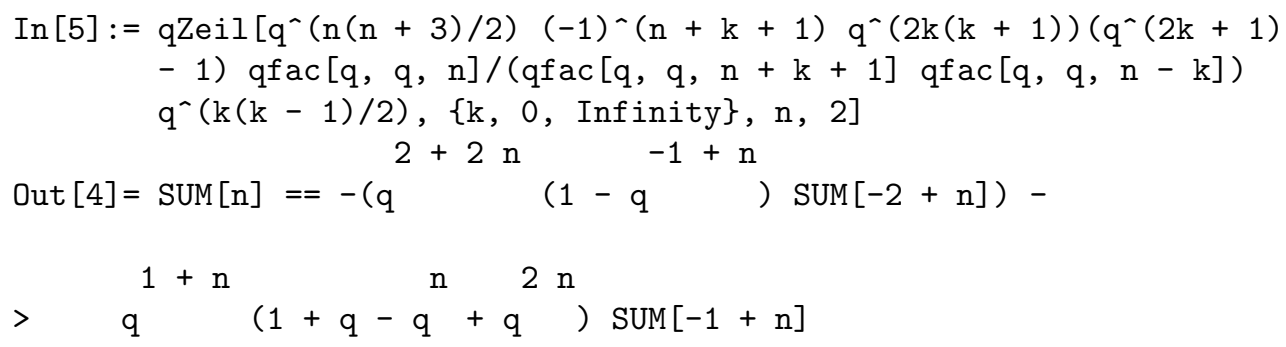

Thus, the program computes not only a recursion relation, but also the order of a minimal one. Experimentally, it follows that $c(p, n)$ satisfies a recursion relation of order $|p|$, for all $p$. Perhaps one can guess the form of a minimal order recursion relation for all twist knots.

Actually, more is true. Namely, the formula for $c(p, n)$ shows that it is a $q-$ holonomic function in both variables $(p, n)$. Thus, we are guaranteed to find 
recursion relations with respect to $n$ and with respect to $p$. Usually, recursion relations with respect to $p$ for fixed $n$ are called skein theory for the $n$th colored Jones function, because the knot is changing, and the color is fixed.

Thus, $q$-holonomicity implies skein relations (with respect to the number of twists) for the $n$th colored Jones polynomial of twist knots, for every fixed $n$.

For computations of recursion relations of the cyclotomic function of twist knots, we refer the reader to 12 .

\subsection{Recursion relations for the colored Jones function of the figure 8 knot}

The Mathematica package qMultiSum.m can compute recursion relations for $q$-multisums. Using this, we can compute equally easily the recursion relation for the colored Jones function. Due to the length of the output, we illustrate this by computing the recursion relation for the colored Jones function of the Figure 8 knot. Recall from Equation (2) for $p=-1$ and from the fact that $c(-1, n)=1$ that the colored Jones function of the figure 8 knot is given by:

$$
J_{K(-1)}(n)=\sum_{k=0}^{\infty} q^{n k}\left(q^{-n-1} ; q^{-1}\right)_{k}\left(q^{-n+1} ; q\right)_{k} .
$$

In computer talk,

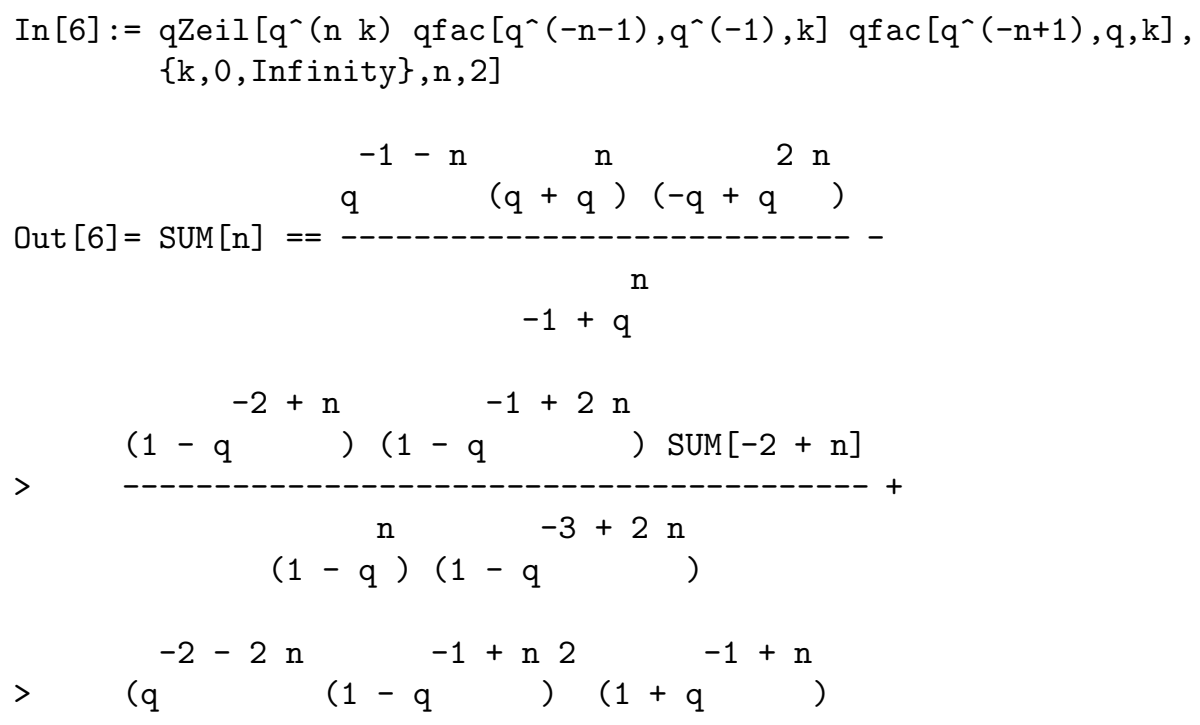




$$
\begin{aligned}
& \left.>\quad\left(q^{4}+q^{4}-q^{3+n}-q^{1+2 n}-q^{3+2 n}-q^{1+3 n}\right) \operatorname{SUM}[-1+n]\right) / \\
& >\quad\left(\left(1-q^{n}(1-q-3+2 n\right.\right.
\end{aligned}
$$

This is a second order inhomogeneous recursion relation for the colored Jones function. A third order homogeneous relation may be obtained by:

$$
\begin{aligned}
& \operatorname{In}[7]:=\text { MakeHomRec }[\%, \operatorname{SUM}[n]]
\end{aligned}
$$

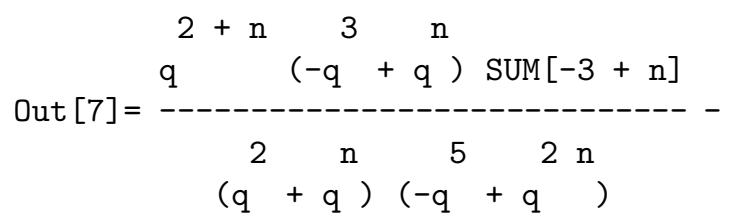

$$
\begin{aligned}
& >\quad\left(q ^ { - 2 - n } ( q ^ { 2 } - q ^ { n } ) \left(q^{8}+q^{4}-2 q^{6+n}+q^{7+n}-q^{2}+2+\right.\right. \\
& \left.\left.>\quad \mathrm{q}^{4+2 \mathrm{n}}-\mathrm{q}^{5+2 \mathrm{n}}+\mathrm{q}^{1+3 \mathrm{n}}-2 \mathrm{q}^{2+3 \mathrm{n}}\right) \operatorname{SUM}[-2+\mathrm{n}]\right) / \\
& >\quad\left(\left(q+q^{n}\right)\left(q^{5}-q^{2 n}\right)\right)+ \\
& >\quad\left(q ^ { - 1 - n } ( - q + q ^ { n } ) \left(q^{4}+q^{4}+q^{2+n}-2 q^{3+n}-q^{1+2 n}+\right.\right. \\
& \left.\left.>\quad \mathrm{q}^{2+2 \mathrm{n}}-\mathrm{q}^{3+2 \mathrm{n}}-2 \mathrm{q}^{1+3 \mathrm{n}}+\mathrm{q}^{2+3 \mathrm{n}}\right) \operatorname{SUM}[-1+\mathrm{n}]\right) /
\end{aligned}
$$

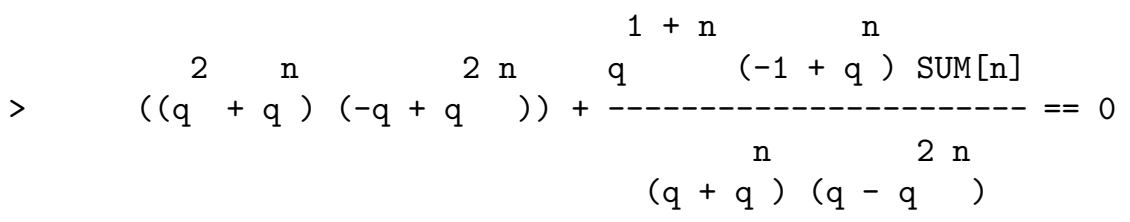

Of course, we can clear denominators and write the above recursion relation using the $q$-Weyl algebra $\mathcal{A}$. Let us end with a matching the theoretical bound for the recursion relation from Section [5 with the computer calculated bound from this section. Using Theorem $[$ it follows that the summand satisfies a recursion relation of order $J^{\star}=1^{2}+1^{2}=2$. This implies that the colored Jones function of the Figure 8 knot satisfies an inhomogeneous relation of degree 2 as was found above. The program also confirms that the colored Jones function 
of the Figure 8 knot does not satisfy an inhomogeneous relation of order less than 2 .

\section{$7 \quad$ The colored Jones function for a simple Lie algebra}

Fix a simple complex Lie algebra $\mathfrak{g}$ of rank $\ell$. For every knot $\mathcal{K}$ and every finite-dimensional $\mathfrak{g}$-module $V$, called the color of the knot, one can define the quantum invariant $J_{\mathcal{K}}(V) \in \mathbb{Z}\left[q^{ \pm 1 / 2 D}\right]$, where $D$ is the determinant of the Cartan matrix of $\mathfrak{g}$. Simple $\mathfrak{g}$-modules are parametrized by the set of dominant weights, which can be identified, after we choose fixed fundamental weights, with $\mathbb{N}^{\ell}$. Hence $J_{\mathcal{K}}$ can be considered as a function $J_{\mathcal{K}}: \mathbb{N}^{\ell} \rightarrow \mathbb{Z}\left[q^{ \pm 1 / 2 D}\right]$.

Theorem 6 For every simple Lie algebra other than $G_{2}$, and a set of fixed fundamental weights, the colored Jones function $J_{\mathcal{K}}: \mathbb{N}^{\ell} \rightarrow \mathbb{Z}\left[q^{ \pm 1 / 2 D}\right]$ is $q-$ holonomic.

Hence the colored Jones function will satisfy some recursion relations, which, together with values at a finitely many initial colors, totally determine the colored Jones function $J_{\mathcal{K}}$.

Remark 7.1 The reason we exclude the $G_{2}$ Lie algebra is technical. Namely, at present we cannot prove that the structure constants of the multiplication of the quantized enveloping algebra of $G_{2}$ with respect to a standard PBW basis,

are $q$-holonomic; see Remark A.3. We believe however, that the theorem also holds for $G_{2}$.

The proof occupies the rest of this section. We will define $J_{\mathcal{K}}$ using representation of the braid groups coming from the $R$-matrix acting on Verma modules (instead of finite-dimensional modules). We then show that the $R$-matrix is $q$-holonomic. The theorem follows from that fact that products and traces of $q$-holonomic matrices are $q$-holonomic.

\subsection{Preliminaries}

Fix a Cartan subalgebra $\mathfrak{h}$ of $\mathfrak{g}$ and a basis $\left\{\alpha_{1}, \ldots, \alpha_{\ell}\right\}$ of simple roots for the dual space $\mathfrak{h}^{*}$. Let $\mathfrak{h}_{\mathbb{R}}^{*}$ be the $\mathbb{R}$-vector space spanned by $\alpha_{1}, \ldots, \alpha_{\ell}$. The root lattice $Y$ is the $\mathbb{Z}$-lattice generated by $\left\{\alpha_{1}, \ldots, \alpha_{\ell}\right\}$. Let $X$ be the weight lattice that is spanned by the fundamental weights $\lambda_{1}, \ldots, \lambda_{\ell}$. Normalize the 
invariant scalar product $(\cdot, \cdot)$ on $\mathfrak{h}_{\mathbb{R}}^{*}$ so that $(\alpha, \alpha)=2$ for every short root $\alpha$. Let $D$ be the determinant of the Cartan matrix, then $(x, y) \in \frac{1}{D} \mathbb{Z}$ for $x, y \in X$.

Let $s_{i}, i=1, \ldots, \ell$, be the reflection along the wall $\alpha_{i}^{\perp}$. The Weyl group $W$ is generated by $s_{i}, i=1 \ldots, \ell$, with the braid relations together with $s_{i}^{2}=1$. A word $w=s_{i_{1}} \ldots s_{i_{r}}$ is reduced if $w$, considered as an element of $W$, can not be expressed by a shorter word. In this case the length $l(w)$ of the element $w \in W$ is $r$. The longest element $\omega_{0}$ in $W$ has length $t=(\operatorname{dim}(\mathfrak{g})-\ell) / 2$, the number of positive roots of $\mathfrak{g}$.

\subsubsection{The quantum group $\mathcal{U}$}

The quantum group $\mathcal{U}=\mathcal{U}_{q}(\mathfrak{g})$ associated to $\mathfrak{g}$ is a Hopf algebra defined over $\mathbb{Q}(v)$, where $v$ is the usual quantum parameter (see [17, 22]). Here our $v$ is the same as $v$ of Lusztig [22] and is equal to $q$ of Jantzen [17, while our $q$ is $v^{2}$. The standard generators of $\mathcal{U}$ are $E_{\alpha}, F_{\alpha}, K_{\alpha}$ for $\alpha \in\left\{\alpha_{1}, \ldots, \alpha_{\ell}\right\}$. For a full set of relations, as well as a good introduction to quantum groups, see [17. Note that all the $K_{\alpha}$ 's commute with each other.

For an element $\gamma \in Y, \gamma=k_{1} \alpha_{1}+\cdots+k_{\ell} \alpha_{\ell}$, let $K_{\gamma}:=K_{\alpha_{1}}^{k_{1}} \ldots K_{\alpha_{1}}^{k_{1}}$.

There is a $Y$-grading on $\mathcal{U}$ defined by $\left|E_{\alpha}\right|=\alpha,\left|F_{\alpha}\right|=-\alpha$, and $\left|K_{\alpha}\right|=0$. If $x$ is homogeneous, then

$$
K_{\gamma} x=v^{(\alpha,|x|)} x K_{\gamma}
$$

Let $\mathcal{U}^{+}$be the subalgebra of $\mathcal{U}$ generated by the $E_{\alpha}, \mathcal{U}^{-}$by the $F_{\alpha}$, and $\mathcal{U}^{0}$ by the $K_{\alpha}$. It is known that the map

$$
\begin{gathered}
\mathcal{U}^{-} \otimes \mathcal{U}^{0} \otimes \mathcal{U}^{+} \rightarrow \mathcal{U} \\
\left(x, x^{\prime}, x^{\prime \prime}\right) \rightarrow x x^{\prime} x^{\prime \prime}
\end{gathered}
$$

is an isomorphism of vector spaces.

\subsubsection{Verma modules and finite dimensional modules}

Let $\lambda \in X$ be a weight. The Verma module $M(\lambda)$ is a $\mathcal{U}$-module with underlying vector space $\mathcal{U}^{-}$and with the action of $\mathcal{U}$ that is uniquely determined by the following condition. Here $\eta$ is the unit of the algebra $\mathcal{U}^{-}$:

$$
\begin{aligned}
E_{\alpha} \cdot \eta & =0 \quad \text { for all } \alpha \\
K_{\alpha} \cdot \eta & =v^{(\alpha, \lambda)} \eta \quad \text { for all } \alpha \\
F_{\alpha} \cdot x & =F_{\alpha} x \quad \text { for all } \quad \alpha \in\left\{\alpha_{1}, \ldots, \alpha_{\ell}\right\}, x \in \mathcal{U}^{-}
\end{aligned}
$$


If $\left(\lambda \mid \alpha_{i}\right)<0$ for all $i=1, \ldots, \ell$ then $M(\lambda)$ is irreducible. On the other hand if $\left(\lambda \mid \alpha_{i}\right) \geq 0$ for all $i=1, \ldots, \ell$ (ie, $\lambda$ is dominant), then $M(\lambda)$ has a unique proper maximal submodule, and the quotient $L(\lambda)$ of $M(\lambda)$ by the proper maximal submodule is a finite dimensional module (of type 1, see [17]). Every finite dimensional module of type 1 of $\mathcal{U}$ is a direct sum of several $L(\lambda)$.

\subsubsection{Quantum braid group action}

For each fundamental root $\alpha \in\left\{\alpha_{1}, \ldots, \alpha_{\ell}\right\}$ there is an algebra automorphism $T_{\alpha}: \mathcal{U} \rightarrow \mathcal{U}$, as described in [17, Chapter 8]. These automorphisms satisfy the following relations, known as the braid relations, or Coxeter moves.

If $(\alpha, \beta)=0$, then $T_{\alpha} T_{\beta}=T_{\beta} T_{\alpha}$.

If $(\alpha, \beta)=-1$, then $T_{\alpha} T_{\beta} T_{\alpha}=T_{\beta} T_{\alpha} T_{\beta}$.

If $(\alpha, \beta)=-2$, then $T_{\alpha} T_{\beta} T_{\alpha} T_{\beta}=T_{\beta} T_{\alpha} T_{\beta} T_{\alpha}$.

If $(\alpha, \beta)=-3$, then $T_{\alpha} T_{\beta} T_{\alpha} T_{\beta} T_{\alpha} T_{\beta}=T_{\beta} T_{\alpha} T_{\beta} T_{\alpha} T_{\beta} T_{\alpha}$.

Note that the Weyl group is generated by $s_{\alpha}$ with exactly the above relations, replacing $T_{\alpha}$ by $s_{\alpha}$, and the extra relations $s_{\alpha}^{2}=1$.

Suppose $w=s_{i_{1}} \ldots s_{i_{r}}$ is a reduced word, one can define

$$
T_{w}:=T_{\alpha_{i_{1}}} \ldots T_{\alpha_{i_{r}}} .
$$

Then $T_{w}$ is well-defined: If $w, w^{\prime}$ are two reduced words of the same element in $W$, then $T_{w}=T_{w^{\prime}}$. This follows from the fact that any two reduced presentations of an element of $W$ are related by a sequence of Coxeter moves.

\subsubsection{Ordering of the roots}

Suppose $w=s_{i_{1}} s_{i_{2}} \ldots s_{i_{t}}$ is a reduced word representing the longest element $\omega_{0}$ of the Weyl group. For $r$ between 1 and $t$ let

$$
\gamma_{r}(w):=s_{i_{1}} s_{i_{2}} \ldots s_{i_{r-1}}\left(\alpha_{i_{r}}\right) .
$$

Then the set $\left\{\gamma_{i}, i=1, \ldots, t\right\}$ is exactly the set of positive roots. We totally order the set of positive roots by $\gamma_{1}<\gamma_{2}<\cdots<\gamma_{t}$. This order depends on the reduced word $w$, and has the following convexity property: If $\beta_{1}, \beta_{2}$ are two positive roots such that $\beta_{1}+\beta_{2}$ is also a root, then $\beta_{1}+\beta_{2}$ is between $\beta_{1}$ and $\beta_{2}$. In particular, the first and the last, $\gamma_{1}$ and $\gamma_{t}$, are always fundamental roots. Conversely, any convex total ordering of the set of positive roots comes from a reduced word representing the longest element of $W$. 


\subsubsection{PBW basis for $\mathcal{U}^{-}, \mathcal{U}^{+}$, and $\mathcal{U}$}

Suppose $w=s_{i_{1}} \ldots s_{i_{t}}$ is a reduced word representing the longest element of $W$. Let us define

$$
\begin{aligned}
e_{r}(w) & =T_{\alpha_{i_{1}}} T_{\alpha_{i_{2}}} \ldots T_{\alpha_{i_{r-1}}}\left(E_{\alpha_{i_{r}}}\right), \\
f_{r}(w) & =T_{\alpha_{i_{1}}} T_{\alpha_{i_{2}}} \ldots T_{\alpha_{i_{r-1}}}\left(F_{\alpha_{i_{r}}}\right) .
\end{aligned}
$$

Then $\left|e_{r}\right|=\gamma_{r}=-\left|f_{r}\right|$. (We drop $w$ if there is no confusion.)

If $\gamma_{r}$ is one of the fundamental roots, $\gamma_{r}=\alpha \in\left\{\alpha_{1}, \ldots, \alpha_{\ell}\right\}$, then $e_{r}(w)=E_{\alpha}$, $f_{r}(w)=F_{\alpha}$ (and do not depend on $\left.w\right)$.

For $t \geq j \geq i \geq 1$ let $\mathcal{U}^{-}[j, i]$ be the vector space spanned by $f_{j}^{n_{j}} f_{j-1}^{n_{j-1}} \ldots f_{i}^{n_{i}}$, for all $n_{j}, n_{j-1}, \ldots, n_{i} \in \mathbb{N}$ and let $\mathcal{U}^{+}[i, j]$ the vector space spanned by $e_{i}^{n_{i}} e_{i+1}^{n_{i+1}} \ldots e_{j}^{n_{j}}$, for all $n_{j}, n_{j-1}, \ldots, n_{i} \in \mathbb{N}$. It is known that $\mathcal{U}^{-}=\mathcal{U}^{-}[t, 1]$ and $\mathcal{U}^{+}=\mathcal{U}^{+}[1, t]$.

For $\mathbf{n}=\left(n_{1}, \ldots, n_{t}\right) \in \mathbb{N}^{t}, \mathbf{j}=\left(j_{1}, \ldots j_{\ell}\right) \in \mathbb{Z}^{\ell}$ and $\mathbf{m}=\left(m_{1}, \ldots, m_{t}\right) \in \mathbb{N}^{t}$ let us define $\mathbf{f}^{\mathbf{n}}, \mathbf{K}^{\mathbf{j}}$ and $\mathbf{e}^{\mathbf{m}}$ by

$$
\mathbf{f}^{\mathbf{n}}(w):=f_{t}^{n_{t}} \ldots f_{1}^{n_{1}}, \quad \mathbf{K}^{\mathbf{j}}:=K_{j_{1} \alpha_{1}} \ldots K_{j_{\ell} \alpha_{\ell}} \quad \mathbf{e}^{\mathbf{n}}(w):=e_{1}^{n_{1}} \ldots e_{t}^{n_{t}} .
$$

Then as vector spaces over $\mathbb{Q}(v) \mathcal{U}^{-}, \mathcal{U}^{+}$and $\mathcal{U}$ have Poincare-Birkhoff-Witt (in short, PBW) basis

$$
\left\{\mathbf{f}^{\mathbf{n}} \mid \mathbf{n} \in \mathbb{N}^{t}\right\}, \quad\left\{\mathbf{e}^{\mathbf{m}} \mid \mathbf{m} \in \mathbb{N}^{t}\right\}, \quad\left\{\mathbf{f}^{\mathbf{n}} \mathbf{K}^{\mathbf{j}} \mathbf{e}^{\mathbf{m}} \mid \mathbf{n}, \mathbf{m} \in \mathbb{N}^{t}, \mathbf{j} \in \mathbb{Z}^{\ell}\right\}
$$

respectively, associated with the reduced word $w$.

In order to simplify notation, we define $S:=\mathbb{N}^{t} \times \mathbb{Z}^{\ell} \times \mathbb{N}^{t}$, and $x_{\sigma}:=\mathbf{f}^{n} K^{\mathbf{j}} \mathbf{e}^{m}$. Thus,

$$
\left\{x_{\sigma} \mid \sigma \in S\right\}
$$

is a PBW basis of $\mathcal{U}$ with respect to the reduced word $w$.

\subsubsection{A commutation rule}

For $x, y \in \mathcal{U}$ homogeneous let us define

$$
[x, y]_{q}:=x y-v^{(|x|,|y|)} y x .
$$

Note that, in general, $[y, x]_{q}$ is not proportional to $[x, y]_{q}$.

An important property of the PBW basis is the following commutation rule, see [18. If $i<j$ then $\left[f_{i}, f_{j}\right]_{q}$ belongs to $\mathcal{U}^{-}[j-1, i+1]$ (which is 0 if $j=i+1$ ). 
It follows that $\mathcal{U}^{-}[j, i]$ is an algebra. This allows us to sort algorithmically noncommutative monomials in the variables $f_{i}$. Also two consecutive variables always $q$-commute: $\left[f_{i}, f_{i+1}\right]_{q}=0$.

Similarly, if $i<j$ then $\left[e_{i}, e_{j}\right]_{q}$ belongs to $\mathcal{U}^{+}[i+1, j-1]$ (which is 0 if $j=i+1$ ). It follows that $\mathcal{U}^{+}[i, j]$ is an algebra, and two consecutive variables always $q^{-}$ commute, $\left[e_{i}, e_{i+1}\right]_{q}=0$.

\section{2 $q$-holonomicity of quantum groups}

Suppose $A: \mathcal{U} \rightarrow \mathcal{U}$ is a linear operator. Using the PBW basis of $\mathcal{U}$ (see Equation (44)), we can present $A$ by a matrix:

$$
A\left(x_{\sigma}\right)=\sum_{\sigma^{\prime}} A_{\sigma}^{\sigma^{\prime}} x_{\sigma^{\prime}}
$$

with $A_{\sigma}^{\sigma^{\prime}} \in \mathbb{Q}(v)$. We call $\left(\sigma, \sigma^{\prime}\right)$ the coordinates of the matrix entry $A_{\sigma}^{\sigma^{\prime}}$.

Definition 7.2 We say that $A$ is $q$-holonomic if the matrix entry $A_{\sigma}^{\sigma^{\prime}}$, considered as a function of $\left(\sigma, \sigma^{\prime}\right)$ is $q$-holonomic with respect to all the variables.

A priori this definition depends on the reduced word $w$. But we will soon see that if $A$ is $q$-holonomic in one PBW basis, then it is so in any other PBW basis.

\subsection{1 $q$-holonomicity of transition matrix}

Suppose $x_{\sigma}\left(w^{\prime}\right)$ is another PBW basis associate to another reduced word $w^{\prime}$ representing the longest element of $W$. Then we have the transition matrix $M_{\sigma}^{\sigma^{\prime}}$ between the two bases, with entries in $\mathbb{Q}(v)$. The next proposition checks that the entries of the transition matrix are $q$-holonomic, by a standard reduction to the rank 2 case.

Proposition 7.3 Except for the case of $G_{2}$, the matrix entry $M_{\sigma}^{\sigma^{\prime}}$ is $q^{-}$ holonomic with respect to all its coordinates.

Proof Since any two reduced presentations of an element of $W$ are related by a sequence of Coxeter moves, it is enough to consider the case of a single Coxeter move. Since each Coxeter move involves only two fundamental roots and all $T_{\alpha}$ 's are algebra isomorphisms, it is enough to considered the case of rank 2 Lie algebras. For all rank 2 Lie algebras (except $G_{2}$ ) we present the proof in Appendix. 


\subsubsection{Structure constants}

Recall the PBW basis $\left\{x_{\sigma} \mid \sigma \in S\right\}$ of the algebra $\mathcal{U}$. The multiplication in $\mathcal{U}$ is determined by the structure constants $c\left(\sigma, \sigma^{\prime}, \sigma^{\prime \prime}\right) \in \mathbb{Q}(v)$ defined by:

$$
x_{\sigma} x_{\sigma^{\prime}}=\sum_{\sigma^{\prime \prime}} c\left(\sigma, \sigma^{\prime}, \sigma^{\prime \prime}\right) x_{\sigma^{\prime \prime}} .
$$

We will show the following:

Theorem 7 The structure constant $c\left(\sigma, \sigma^{\prime}, \sigma^{\prime \prime}\right)$ is $q$-holonomic with respect to all its variables.

Proof will be given in subsection 7.4 .5

\subsubsection{Actions on Verma modules are $q$-holonomic}

Each Verma module $M(\lambda)$ is naturally isomorphic to $\mathcal{U}^{-}$, as a vector space, via the map $u \rightarrow u \cdot \eta$. Using this isomorphism we identify a PBW basis of $\mathcal{U}^{-}$ with a basis of $M(\lambda)$, also called a PBW basis. If $u \in \mathcal{U}$, then the action of $u$ on $M(\lambda)$ in a PBW basis can be written by a matrix $u_{\mathbf{n}}^{\mathbf{n}^{\prime}}$ with entries in $\mathbb{Q}(v)$. We call $\left(\mathbf{n}, \mathbf{n}^{\prime}\right) \in \mathbb{N}^{t} \times \mathbb{N}^{t}$ the coordinates of the matrix entry.

Proposition 7.4 For every $r$ with $1 \leq r \leq t$, the entries of the matrices $e_{r}^{k}, f_{r}^{k}$ are $q$-holonomic with respect to $k, \lambda$, and the coordinates of the entry.

This Proposition follows immediately from Theorem 7 and Fact 0.

\subsection{Quantum knot invariants}

\subsubsection{The quasi- $R$-matrix}

Fix a reduced word $w$ representing the longest element of $W$. For each $r, 1 \leq$ $r \leq t$, let

$$
\Theta_{r}:=\sum_{k \in \mathbb{N}} c_{k} f_{r}^{k} \otimes e_{r}^{k}
$$

where

$$
c_{k}=(-1)^{k} v_{\gamma_{r}}^{-k(k-1) / 2} \frac{\left(v_{\gamma_{r}}-v_{\gamma_{r}}^{-1}\right)^{k}}{[k]_{\gamma_{r}} !} .
$$


Here $v_{\gamma}=v^{(\gamma \mid \gamma) / 2}$, and

$$
[k]_{\gamma} !=\prod_{i=1}^{k} \frac{v_{\gamma}^{i}-v_{\gamma}^{-i}}{v_{\gamma}-v_{\gamma}^{-1}} .
$$

The main thing to observe is that $c_{k}$ is $q$-holonomic with respect to $k$. Note that although $\Theta_{r}$ is an infinite sum, for every weight $\lambda \in X$, the action of $\Theta_{r}$ on $M(\lambda) \otimes M(\lambda)$ is well-defined. This is because the action of $e_{r}$ is locally nilpotent, ie, for every $x \in M(\lambda)$, there is $k$ such that $e_{r}^{k} \cdot x=0$.

The quasi-R-matrix is:

$$
\Theta:=\Theta_{t} \Theta_{t-1} \ldots \Theta_{1} .
$$

We will consider $\Theta$ as an operator from $M(\lambda) \otimes M(\lambda)$ to itself. There is a natural basis for $M(\lambda) \otimes M(\lambda)$ coming from the PBW basis of $M(\lambda)$.

Proposition 7.5 The matrix of $\Theta$ acting on $M(\lambda)$ in a $P B W$ basis is $q-$ holonomic with respect to all the coordinates of the entry and $\lambda$.

Proof It's enough to prove the statement for each $\Theta_{r}$. The result for $\Theta_{r}$ follows from the fact that the actions of $e_{r}^{k}, f_{r}^{k}$ on $M(\lambda)$, as well as $c_{k}$, are $q$-holonomic in $k$ and so are all the coordinates of the matrix entries, by Proposition 7.4 .

\subsubsection{The $R$-matrix and the braiding}

As usual, let us define the weight on $M(\lambda)$ by declaring the weight of $F_{\mathbf{n}} \cdot \eta$ to be $\lambda-\sum n_{i} \gamma_{i}$, where $\mathbf{n}=\left(n_{1}, \ldots, n_{t}\right)$. The space $M(\lambda)$ is the direct sum of its weight subspaces.

Let $\mathcal{D}: M(\lambda) \otimes M(\lambda) \rightarrow M(\lambda) \otimes M(\lambda)$ be the linear operator defined by

$$
\mathcal{D}(x \otimes y)=v^{-(|x|,|y|)} x \otimes y .
$$

Clearly $\mathcal{D}$ is $q$-holonomic; it's called the diagonal part of the $R$-matrix, which is $R:=\Theta \mathcal{D}$.

The braiding is $\mathcal{B}:=R \sigma$, where $\sigma(x \otimes y)=y \otimes x$. Combining the above results, we get the following:

Theorem 8 The entry of the matrix of the braiding acting on $M(\lambda)$ is $q-$ holonomic with respect to all the coordinates and $\lambda$.

Remark 7.6 Technically, in order to define the diagonal part $\mathcal{D}$, one needs to extend the ground ring to include a $D$-th root of $v$, since $(\lambda, \mu)$, with $\lambda, \mu \in X$, is in general not an integer, but belonging to $\frac{1}{D} \mathbb{Z}$. 


\subsection{3 $q$-holonomicity of quantum invariants of knots}

First let us recall the definition of quantum knot invariant.

Using the braiding $\mathcal{B}: M(\lambda) \rightarrow M(\lambda)$ one can define a representation of the braid group $\tau: B_{m} \rightarrow(M(\lambda))^{\otimes m}$ by putting

$$
\tau\left(\sigma_{i}\right):=\mathrm{id}^{\otimes i-1} \otimes \mathcal{B} \otimes \mathrm{id}^{\otimes m-i-1} .
$$

Let $\rho$ denote the half-sum of positive roots. For an element $x \in \mathcal{U}$ and an $\mathcal{U}$-module $V$, the quantum trace is defined as

$$
\operatorname{tr}_{q}(x, V):=\operatorname{tr}\left(x K_{-2 \rho}, V\right) .
$$

Suppose a framed knot $\mathcal{K}$ is obtained by closing a braid $\beta \in B_{m}$. We would say that the colored Jones polynomial is the quantum trace of $\tau(\beta)$. However, since $M(\lambda)$ is infinite-dimensional, the trace may not make sense. Instead, we will use a trick of breaking the knot. Let $\mathcal{K}^{\prime}$ denote the long knot which is the closure of all but the first strand of $\beta$.

Recall that $\tau(\beta)$ acts on $(M(\lambda))^{\otimes m}$. Let

$$
\tau(\beta)(\lambda)_{\mathbf{n}_{1}, \ldots, \mathbf{n}_{m}}^{\mathbf{n}_{1}^{\prime}, \ldots, n_{m}^{\prime}} \in \mathbb{Z}\left[v^{ \pm 1 / D}\right]
$$

be the entries of the matrix $\tau(\beta)(\lambda)$. We will take partial trace by first putting $\mathbf{n}_{1}=\mathbf{n}_{1}^{\prime}=0$ and then take the sum over all $\mathbf{n}_{2}=\mathbf{n}_{2}^{\prime}, \ldots, \mathbf{n}_{m}=\mathbf{n}_{m}^{\prime}$. The following lemma shows that the sum is actually finite.

Lemma 7.7 Suppose $\mathbf{n}_{1}=0$. There are only a finite number of collections of $\left(\mathbf{n}_{2}, \mathbf{n}_{3}, \ldots, \mathbf{n}_{m}\right) \in \mathbb{N}^{t-1}$ such that

$$
\tau(\beta)(\lambda)_{\mathbf{n}_{1}, \ldots, \mathbf{n}_{m}}^{\mathbf{n}_{1}, \ldots, \mathbf{n}_{m}}
$$

is not zero.

Proof Let $M^{\prime}(\lambda)$ be the maximal proper $\mathcal{U}$-submodule of $M(\lambda)$. Then $L(\lambda)=M(\lambda) / M^{\prime}(\lambda)$ is a finite dimensional vector space. In particular it has only a finite number of non-trivial weights. Hence, all except for a finite number of $\mathbf{f}_{\mathbf{n}}, \mathbf{n} \in \mathbb{N}^{t}$, are in $M^{\prime}(\lambda)$.

We present the coefficients $\mathcal{B}_{ \pm}(\lambda)$ graphically as in Figure 3

Note that if $\left(\mathcal{B}_{ \pm}\right)_{\mathbf{n}_{1}, \mathbf{n}_{2}}^{\mathbf{m}_{1}, \mathbf{m}_{2}}$ is not equal to 0 , then $\mathbf{f}_{\mathbf{m}_{2}}$ can be obtained from $\mathbf{f}_{\mathbf{n}_{1}}$ by action of an element in $\mathcal{U}$, and similarly, $\mathbf{f}_{\mathbf{m}_{1}}$ can be obtained from $\mathbf{f}_{\mathbf{n}_{2}}$ by action of an element in $\mathcal{U}$. Thus if we move upwards along a string of the braid, 


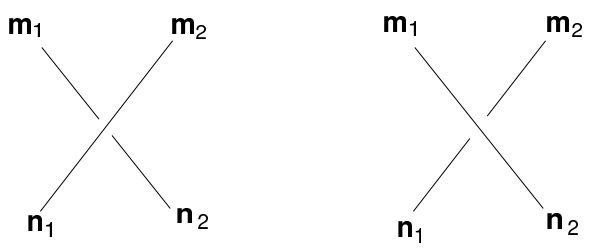

Figure 3: $\left(\mathcal{B}_{+}\right)_{\mathbf{n}_{1}, \mathbf{n}_{2}}^{\mathbf{m}_{1}, \mathbf{m}_{2}}$ and $\left(\mathcal{B}_{-}\right)_{\mathbf{n}_{1}, \mathbf{n}_{2}}^{\mathbf{m}_{1}, \mathbf{m}_{2}}$

the basis element at the top can always be obtained from the one at the bottom by an action of $\mathcal{U}$.

Because the closure of $\beta$ is a knot, by moving around the braid one can get any point from any particular point. Because the basis element $\mathbf{f}_{0}$ is not in $M^{\prime}(\lambda)$, we conclude that if

$$
\tau(\beta)(\lambda)_{\mathbf{n}_{1}, \ldots, \mathbf{n}_{m}}^{\mathbf{n}_{1}, \ldots, \mathbf{n}_{m}}
$$

is not 0 , with $\mathbf{n}_{1}=0$, then all the basis vectors $\mathbf{f}_{\mathbf{n}_{2}}, \ldots, \mathbf{f}_{\mathbf{n}_{m}}$ are not in $M^{\prime}(\lambda)$, and there are only a finite number of such collections.

$\mathrm{R}$ ecall that $2 \rho$ is the sum of all positive roots. Let us define

$$
J_{\mathcal{K}^{\prime}}(\lambda)=\sum_{\mathbf{n}_{2}, \ldots \mathbf{n}_{m} \in \mathbb{N}^{t}, \mathbf{n}_{1}=0}\left(K_{-2 \rho} \tau(\beta)(\lambda)\right)_{\mathbf{n}_{1}, \ldots, \mathbf{n}_{m}}^{\mathbf{n}_{1}, \ldots, n_{m}} .
$$

From $q$-holonomicity of $\tau(\beta)(\lambda)$ it follows that $J_{\mathcal{K}^{\prime}}(\lambda)$ is $q$-holonomic. $J_{\mathcal{K}^{\prime}}(\lambda)$ is a long knot invariant, and is related to the colored Jones polynomial $J_{\mathcal{K}}$ of the knot $\mathcal{K}$ by

$$
J_{\mathcal{K}}(\lambda)=J_{\mathcal{K}^{\prime}}(\lambda) \times \operatorname{dim}_{q}(L(\lambda)),
$$

where $L(\lambda)$ is the finite-dimensional simple $\mathcal{U}$-module of highest weight $\lambda$, and $\operatorname{dim}_{q}(L(\lambda))$ is its quantum dimension, and is given by the formula

$$
\operatorname{dim}_{q}(L(\lambda))=\prod_{\alpha>0} \frac{v^{(\lambda+\rho, \alpha)}-v^{-(\lambda+\rho, \alpha)}}{v^{(\rho, \alpha)}-v^{-(\rho, \alpha)}} .
$$

Since $\operatorname{dim}_{q}(L(\lambda))$ is $q$-holonomic in $\lambda$, we see that $J_{\mathcal{K}}(\lambda)$ is $q$-holonomic. This completes the proof of Theorem 6 .

Remark 7.8 The invariant $J_{\mathcal{K}^{\prime}}$ of long knots is sometime more convenient. For example, $J_{\mathcal{K}}(\lambda)$ might contain fractional power of $q$, but (if $\mathcal{K}^{\prime}$ has framing 0,) J_{\mathcal{K}^{\prime}}(\lambda)$ is always in $\mathbb{Z}\left[q^{ \pm 1}\right]$, see [20]. Also the function $J_{\mathcal{K}^{\prime}}$ can be extended to the whole weight lattice. 


\subsection{Proof of Theorem 7}

\subsection{1 $\quad r_{\alpha}$ is $q$-holonomic}

We will need the linear maps $r_{\alpha}, r_{\alpha}^{\prime}: \mathcal{U}^{ \pm} \rightarrow \mathcal{U}^{ \pm}$, as defined in [17, Chapter 6]. Their restriction to $\mathcal{U}^{-}$is uniquely characterized by the properties:

$$
r_{\alpha}(x y)=r_{\alpha}(x) y+v^{(\alpha,|x|)} x r_{\alpha}(y) \quad r_{\alpha}^{\prime}(x y)=x r_{\alpha}^{\prime}(y)+v^{(\alpha,|x|)} r_{\alpha}^{\prime}(x) y
$$

and for any two fundamental roots $\alpha, \beta$, (see [17. Eqn.(6.15.4)]) and

$$
r_{\alpha}\left(F_{\beta}^{n}\right)=r_{\alpha}^{\prime}\left(F_{\beta}^{n}\right)=\delta_{\alpha, \beta} \frac{1-v_{\alpha}^{2 n}}{1-v_{\alpha}^{2}} F_{\alpha}^{n-1},
$$

where $v_{\alpha}:=v^{(\alpha, \alpha) / 2} ;$ see [17, Eqn.(8.26.2)].

Lemma 7.9 For a fixed $\alpha \in\left\{\alpha_{1}, \ldots, \alpha_{\ell}\right\}$, the matrix entries of the operators $\left(r_{\alpha}\right)^{k},\left(r_{\alpha}^{\prime}\right)^{k}: \mathcal{U}^{-} \rightarrow \mathcal{U}^{-}$are $q$-holonomic with respect to $k$ and the coordinates of the matrix entry. Similarly, $\left(r_{\alpha}\right)^{k},\left(r_{\alpha}^{\prime}\right)^{k}: \mathcal{U}^{+} \rightarrow \mathcal{U}^{+}$are $q$-holonomic.

Proof We give a proof for $r_{\alpha}^{k}: \mathcal{U}^{-} \rightarrow \mathcal{U}^{-}$. The other case is similar.

There is a reduced word $w^{\prime}=s_{i_{1}} \ldots s_{i_{t}}$ representing the longest element $\omega_{0}$ of $W$ such that $\alpha_{i_{1}}=\alpha$. Then $w=s_{i_{2}} \ldots s_{i_{t}} s_{\bar{\alpha}}$ is another reduced word representing $\omega_{0}$, where $\bar{\alpha}:=-\omega_{0}(\alpha)$.

For the PBW basis of $\mathcal{U}^{-}$associated with $w$ it's known that $\gamma_{t}=\alpha$, and thus $f_{t}=F_{\alpha}$. According to [17, 8.26.5], for every $x$ in the algebra $\mathcal{U}^{-}[t-1,1]$, one has

$$
r_{\alpha}(x)=0 .
$$

Using Equations (5) and (6) and induction, one can easily show that for every $x \in \mathcal{U}^{-}[t-1,1]$,

$$
\left(r_{\alpha}\right)^{k}\left(f_{t}^{n_{t}} x\right)=\prod_{i=1}^{k} \frac{1-v_{\alpha}^{2 n_{t}-2 i+2}}{1-v_{\alpha}^{2}} f_{t}^{n_{t}-k} x,
$$

This formula, applied to $x=f_{t-1}^{n_{t-1}} \ldots f_{1}^{n_{1}}$, proves the statement.

\subsection{2 $U_{q}\left(\mathfrak{s l}_{2}\right)$ is $q$-holonomic}

Lemma 7.10 Theorem $\square$ holds true for $\mathfrak{g}=\mathfrak{s l}_{2}$. 
Proof The PBW basis for $\mathcal{U}$ is $F^{n} K^{j} E^{m}$, with $m, n \in \mathbb{N}$ and $j \in \mathbb{Z}$. First of all we know that

$$
E_{\alpha}^{m} F_{\alpha}^{n}=\sum_{i=0}^{\infty}\left[\begin{array}{c}
m \\
i
\end{array}\right]_{v_{\alpha}}\left[\begin{array}{c}
n \\
i
\end{array}\right]_{v_{\alpha}} F_{\alpha}^{n-i} b\left(K_{\alpha} ; 2 i-n-m, i\right) E_{\alpha}^{m-i},
$$

where

$$
b\left(K_{\alpha} ; a, i\right):=\prod_{j=1}^{i} \frac{K_{\alpha} v_{\alpha}^{a-j+1}-K_{\alpha}^{-1} v_{\alpha}^{-a+j-1}}{v_{\alpha}-v_{\alpha}^{-1}} .
$$

Here for any root $\gamma$, one defines $v_{\gamma}=v^{(\gamma, \gamma) / 2}$, and $\left[\begin{array}{c}m \\ i\end{array}\right]_{v_{\alpha}}$ is the usual quantum binomial coefficient calculated with $v$ replaced by $v_{\alpha}$.

Hence

$$
\left(F^{m} K^{k} E^{n}\right)\left(F^{m^{\prime}} K^{k^{\prime}} E^{m^{\prime}}\right)=\sum_{i=0}^{\infty} F^{m+m^{\prime}-i} a\left(m, k, n, m^{\prime}, k^{\prime}, n^{\prime}, i\right) E^{n+n^{\prime}-i},
$$

where

$$
\begin{aligned}
& a\left(m, k, n, m^{\prime}, k^{\prime}, n^{\prime}, i\right) \\
& \quad=v^{2 k\left(i-m^{\prime}\right)+2 k^{\prime}(i-n)}\left[\begin{array}{c}
n \\
i
\end{array}\right]\left[\begin{array}{c}
m^{\prime} \\
i
\end{array}\right][i] ! b\left(K ; 2 i-n-m^{\prime}, i\right) K^{k+k^{\prime}} .
\end{aligned}
$$

The value of the function $a$ is in $\mathbb{Z}\left[v^{ \pm 1}\right]\left[K^{ \pm 1}\right]$. Consider the coefficient of $K^{r}$ in $a$; one gets a function of $m, n, k, m^{\prime}, n^{\prime}, k^{\prime}, i, r$ with values in $\mathbb{Z}\left[v^{ \pm 1}\right]$ which is clearly $q$-holonomic with respect to all variables. The lemma follows.

\subsection{3 $E_{\alpha}^{k}, F_{\alpha}^{k}: \mathcal{U} \rightarrow \mathcal{U}$ are $q-$ holonomic in $k$}

Proposition 7.11 For a fixed fundamental root $\alpha \in\left\{\alpha_{1}, \ldots, \alpha_{\ell}\right\}$, the operators $E_{\alpha}^{k}, F_{\alpha}^{k}: \mathcal{U} \rightarrow \mathcal{U}$ of left multiplication are $q$-holonomic with respect to $k$ and all the coordinates of the matrix entry. Similarly, the right multiplication by $E_{\alpha}^{k}, F_{\alpha}^{k}$ are $q$-holonomic with respect to $k$ and all the coordinates of the matrix entry.

Proof (a) Left multiplication by $F_{\alpha}^{k}$ and right multiplication by $E_{\alpha}^{k}$.

Choose $w$ as in the proof of Lemma 7.9. Then $f_{t}=F_{\alpha}$ and $e_{t}=E_{\alpha}$, and an element of the PBW basis has the form $f_{t}^{n_{t}} x K_{\beta} y e_{t}^{m_{t}}$. It's clear that left multiplication by $F_{\alpha}$ and right multiplication by $E_{\alpha}$ are $q$-holonomic.

(b) Left multiplication by $E_{\alpha}^{k}$. 
Choose a reduced word $w=s_{i_{1}} \ldots s_{i_{t}}$ representing the longest element $\omega_{0}$ that begins with $\alpha$ : $\alpha_{i_{1}}=\alpha$. We have the corresponding PBW basis $f_{i}, e_{i}, i=$ $1, \ldots, t$ with $f_{1}=F_{\alpha}$ and $e_{1}=E_{\alpha}$. Thus a typical element of the PBW basis has the form

$$
x F_{\alpha}^{n_{1}} K_{\beta} E_{\alpha}^{m_{1}} y
$$

where $x=f_{t}^{n_{t}} \ldots f_{2}^{n_{2}}, y=e_{2}^{m_{2}} \ldots e_{t}^{m_{t}}$. By [17, 8.26.6], since $x \in \mathcal{U}^{-}[t, 2]$, one has $r_{\alpha}^{\prime}(x)=0$. Using formula [17, 6.17.1], one can easily prove by induction that

$$
\left(E_{\alpha}\right)^{k} x=\sum_{i=0}^{\infty} v^{i-i k}\left[\begin{array}{c}
k \\
i
\end{array}\right]_{v_{\alpha}} \frac{K_{\alpha}^{i}}{\left(v_{\alpha}-v_{\alpha}^{-1}\right)^{i}}\left(r_{\alpha}\right)^{i}(x) E_{\alpha}^{k-i} .
$$

Using this formula one can move the $E_{\alpha}$ past $x$ in the expression (7), (there appear $r_{\alpha}$ and $K_{\alpha}$ ), then one moves $E_{\alpha}$ past $F_{\alpha}$ using the $\mathfrak{s l}_{2}$ case. The last step is moving past $K_{\beta}$ is easy, since

$$
E_{\alpha} K_{\beta}=v^{-(\beta, \alpha)} K_{\beta} E_{\alpha} .
$$

Using Lemmas $[7.9$ and 7.10, we see that each "moving step" is $q$-holonomic. Hence we get the result for the left multiplication by $E_{\alpha}^{k}$.

(c) Right multiplication by $F_{\alpha}^{k}$.

The proof is similar. We use the same basis (7) as in the case b). For $y$, by Lemma 8.26 of [17], one has $r_{\alpha}(y)=0$. Hence using induction based on the formula (6.17.2) of [17] one can show that

$$
y F_{\alpha}^{n}=\sum_{i=0}^{\infty} \frac{v_{\alpha}^{i(n-i)}}{\left(v_{\alpha}^{-1}-v_{\alpha}\right)^{i}}\left[\begin{array}{c}
n \\
i
\end{array}\right]_{v_{\alpha}} F_{\alpha}^{n-i} K_{\alpha}^{-i}\left(r_{\alpha}^{\prime}\right)^{i}(y) .
$$

Using this formula, and the results for $r_{\alpha}^{\prime}$ (Lemma 7.9) and $\mathfrak{s l}_{2}$ (Lemma 7.10) we can move $F_{\alpha}$ to the right.

\subsection{4 $T_{\alpha}$ is $q$-holonomic}

Proposition 7.12 For a fixed fundamental root $\alpha \in\left\{\alpha_{1}, \ldots, \alpha_{\ell}\right\}$, the braid operator $T_{\alpha}: \mathcal{U} \rightarrow \mathcal{U}$ and its inverse $T_{\alpha}^{-1}$ are $q$-holonomic.

Proof By Proposition 7.3 we can use any PBW basis.

Choose a reduced word $w^{\prime}=s_{i_{1}} \ldots s_{i_{t}}$ representing the longest element $\omega_{0}$ that begins with $\alpha: \alpha_{i_{1}}=\alpha$. Then $w=s_{i_{2}} \ldots s_{i_{t}} s_{\bar{\alpha}}$ is another reduced word representing $\omega_{0}$, where $\bar{\alpha}$ is the dual of $\alpha: \bar{\alpha}=-\omega_{0}(\alpha)$. 
We use $f_{r}$ to denote $f_{r}(w)$, and $f_{r}^{\prime}$ to denote $f_{r}\left(w^{\prime}\right)$. The relation between the two PBW basis of $w$ and $w$ is as follows: For $1 \leq r \leq t-1$,

$$
T_{\alpha}\left(f_{r}\right)=f_{r+1}^{\prime}, \quad T_{\alpha}\left(e_{r}\right)=e_{r+1}^{\prime} .
$$

Besides, $f_{t}=F_{\alpha}=f_{1}^{\prime}, e_{t}=E_{\alpha}=e_{1}^{\prime}$.

We will consider the matrix entry of $T_{\alpha}: \mathcal{U} \rightarrow \mathcal{U}$ where the source space is equipped with the PBW corresponding to $w$, while the target space with the PBW basis corresponding to $w^{\prime}$.

From [17. Chapter 8], recall that:

$$
T_{\alpha}\left(F_{\alpha}\right)=-K_{\alpha}^{-1} E_{\alpha}, \quad T_{\alpha}\left(E_{\alpha}\right)=-F_{\alpha} K_{\alpha} .
$$

Hence

$$
T_{\alpha}\left(F_{\alpha}^{n}\right)=(-1)^{n} v_{\alpha}^{n(n-1)} K_{\alpha}^{-n} E_{\alpha}^{n}, \quad T_{\alpha}\left(E_{\alpha}^{m}\right)=(-1)^{m} v_{\alpha}^{-m(m-1)} F_{\alpha}^{m} K_{\alpha}^{m} .
$$

For a basis element $x_{\sigma}=f_{t}^{n_{t}} \ldots f_{1}^{n_{1}} K_{\beta} e_{1}^{m_{1}} \ldots e_{t}^{m_{t}}$, we have

$$
\begin{aligned}
& T_{\alpha}\left(x_{\sigma}\right)=d_{\alpha}\left(n_{t}, m_{t}\right) K_{\alpha}^{-n_{t}} E_{\alpha}^{n_{t}} \times\left(f_{t}^{\prime}\right)^{m_{t-1}} \\
& \ldots\left(f_{1}^{\prime}\right)^{n_{2}} K_{s_{\alpha} \beta}\left(e_{1}^{\prime}\right)^{m_{2}} \ldots\left(e_{t}^{\prime}\right)^{m_{t-1}} \times F_{\alpha}^{m_{t}} K_{\alpha}^{m_{t}},
\end{aligned}
$$

where $\quad d_{\alpha}\left(n_{t}, m_{t}\right):=(-1)^{n_{t}+m_{t}} v_{\alpha}^{n_{t}\left(n_{t}-1\right)-m_{t}\left(m_{1}-1\right)}$.

The left or right multiplication by $K_{\alpha}^{n}$ is $q$-holonomic with respect to $n$ and all the coordinates. The left multiplication by $E^{n_{t}}$, as well as the right multiplication my $F_{\alpha}^{m_{t}}$ is $q$-holonomic with respect to $n_{t}$ and all coordinates, by Proposition [7.11. One then can conclude that $T_{\alpha}$ is $q$-holonomic.

The proof for $T_{\alpha}^{-1}: \mathcal{U} \rightarrow \mathcal{U}$ is similar. One should use the PBW basis of $w^{\prime}$ for the source, and that of $w$ for the target.

\subsubsection{Proof of Theorem 7}

It is clear that for each $\mathbf{j} \in \mathbb{Z}^{\ell}$, the operator $\mathbf{K}^{\mathbf{j}}: \mathcal{U} \rightarrow \mathcal{U}$ of left multiplication is $q$-holonomic.

Fix a reduced word $w$ representing the longest element of $W$. It suffices to show that for each $1 \leq r \leq t$ the operators $e_{r}^{k}, f_{r}^{k}: \mathcal{U} \rightarrow \mathcal{U}$ (left multiplication) are $q$-holonomic with respect to all variables, including $k$.

This is true if $e_{r}=E_{\alpha}$ and $f_{r}=F_{\alpha}$, where $\alpha$ is one of the fundamental roots, by Proposition 17.11. But any $e_{r}$ or $f_{r}$ can be obtained from $E_{\alpha}$ and $F_{\alpha}$ by actions of product of various $T_{\alpha_{i}}$ 's. Hence from Proposition 7.12 we get Theorem [7. 


\section{A Appendix: Proof of Proposition 7.3 for $A_{2}$ and $B_{2}$}

In this appendix we will prove Proposition 7.3 for the rank 2 Lie algebras $A_{2}$ and $B_{2}$. We will achieve this by a brute-force calculation.

First, let us discuss some simplification, due to symmetry. The transition matrix of $\mathcal{U}$ leaves invariant each of $\mathcal{U}^{+}, \mathcal{U}^{-}, \mathcal{U}^{0}$. On $\mathcal{U}^{0}$ the transition matrix is identity. Hence it's enough to consider the restriction of the transition matrix in $\mathcal{U}^{-}$and $\mathcal{U}^{+}$. Furthermore, the Cartan symmetry (the operator $\tau$ of [17]) reduces the case of $\mathcal{U}^{+}$to that of $\mathcal{U}^{-}$.

\section{A.1 The case of $A_{2}$}

There are two fundamental roots denoted by $\alpha$ and $\beta$. The set of positive roots is $\{\alpha, \beta, \alpha+\beta\}$. The reduced representations of the longest element of the Weyl group are $w=s_{1} s_{2} s_{1}$ and $w^{\prime}=s_{2} s_{1} s_{2}$, where $s_{1}=s_{\alpha}$ and $s_{2}=s_{\beta}$.

The total ordering (see Section 7.1.4) of the set of positive roots corresponding to $w$ and $w^{\prime}$ are, respectively:

$$
\begin{aligned}
\left(\gamma_{1}, \gamma_{2}, \gamma_{3}\right) & =(\alpha, \alpha+\beta, \beta) \\
\left(\gamma_{1^{\prime}}, \gamma_{2^{\prime}}, \gamma_{3^{\prime}}\right) & =(\beta, \alpha+\beta, \alpha) .
\end{aligned}
$$

Notice that $\gamma_{i^{\prime}}=\gamma_{3-i}$ for $i=1, \ldots, 3$.

The PBW basis of $\mathcal{U}^{-}$(see Section 17.1.5) corresponding to $w$ and $w^{\prime}$ are, respectively:

$$
\left\{f_{3}^{m} f_{2}^{n} f_{1}^{p} \mid m, n, p \in \mathbb{N}\right\}, \quad\left\{f_{3^{\prime}}^{m} f_{2^{\prime}}^{n} f_{1^{\prime}}^{p} \mid m, n, p \in \mathbb{N}\right\},
$$

where

$$
\begin{aligned}
\left(f_{3}, f_{2}, f_{1}\right) & =\left(F_{\beta}, T_{\alpha}\left(F_{\beta}\right)=-v\left[F_{\beta}, F_{\alpha}\right]_{q}=F_{\beta} F_{\alpha}-v F_{\alpha} F_{\beta}, F_{\alpha}\right) \\
\left(f_{3^{\prime}}, f_{2^{\prime}}, f_{1^{\prime}}\right) & =\left(F_{\alpha}, T_{\beta}\left(F_{\alpha}\right)=F_{\alpha} F_{\beta}-v F_{\beta} F_{\alpha}, F_{\beta}\right) .
\end{aligned}
$$

From explicit formulas of [23, section 5] it follows that:

Lemma A.1 The structure constants of $\mathcal{U}^{-}$, in the basis of $w$, is $q$-holonomic.

Let us define a scalar product $(\cdot, \cdot)$ on $\mathcal{U}^{-}$such that the PBW basis of $w$ is an orthonormal basis. Since

$$
f_{3^{\prime}}^{m^{\prime}} f_{2^{\prime}}^{n^{\prime}} f_{1^{\prime}}^{p^{\prime}}=\sum_{m, n, p}\left(f_{3^{\prime}}^{m^{\prime}} f_{2^{\prime}}^{n^{\prime}} f_{1^{\prime}}^{p^{\prime}}, f_{3}^{m} f_{2}^{n} f_{1}^{n}\right) f_{3}^{m} f_{2}^{n} f_{1}^{p}
$$


Proposition 7.3 is equivalent to showing that

$$
\left(f_{3^{\prime}}^{m^{\prime}} f_{2^{\prime}}^{n^{\prime}} f_{1^{\prime}}^{p^{\prime}}, f_{3}^{m} f_{2}^{n} f_{1}^{n}\right)
$$

is $q$-holonomic in all variables $m, n, p, m^{\prime}, n^{\prime}, p^{\prime}$.

Since multiplication is $q$-holonomic in the PBW basis of $w$ (see Lemma A.1), it suffices to show that

$$
\left(f_{i^{\prime}}^{k}, f_{3}^{m} f_{2}^{n} f_{1}^{n}\right)
$$

is $q$-holonomic in $k, m, n, p$ for each $i=1,2,3$. This is clear for $i=1$ or $i=3$, since $f_{1^{\prime}}=f_{3}$ and $f_{3^{\prime}}=f_{1}$. As for $f_{2^{\prime}}$, an easy induction shows that

$$
f_{2^{\prime}}^{n}=(-v)^{-n} \sum_{k=0}^{\infty} v^{-k(k-3) / 2}\left(v-v^{-1}\right)^{k}\left[\begin{array}{l}
n \\
k
\end{array}\right] f_{3}^{k} f_{2}^{n-k} f_{1}^{k} .
$$

and the statement also holds true for $i=2$. This proves Proposition 7.3 for $A_{2}$.

\section{A.2 The case of $B_{2}$}

There are two fundamental roots denoted here by $\alpha$ and $\beta$, where $\alpha$ is the short root. The set of positive roots is $\{\alpha, \beta, 2 \alpha+\beta, \alpha+\beta\}$. The reduced representations of the longest element of the Weyl group are $w=s_{1} s_{2} s_{1} s_{2}$ and $w^{\prime}=s_{2} s_{1} s_{2} s_{1}$, where $s_{1}=s_{\alpha}$ and $s_{2}=s_{\beta}$.

The total ordering of the set of positive roots corresponding to $w$ and $w^{\prime}$ are, respectively:

$$
\begin{aligned}
\left(\gamma_{1}, \gamma_{2}, \gamma_{3}, \gamma_{4}\right) & =(\alpha, 2 \alpha+\beta, \alpha+\beta, \beta) \\
\left(\gamma_{1^{\prime}}, \gamma_{2^{\prime}}, \gamma_{3^{\prime}}, \gamma_{4^{\prime}}\right) & =(\beta, \alpha+\beta, 2 \alpha+\beta, \alpha) .
\end{aligned}
$$

Notice that $\gamma_{i^{\prime}}=\gamma_{4-i}$ for $i=1, \ldots, 4$.

The PBW basis of $\mathcal{U}^{-}$(see Section [7.1.5) corresponding to $w$ and $w^{\prime}$ are, respectively:

$$
\left\{f_{4}^{l} f_{3}^{m} f_{2}^{n} f_{1}^{p} \mid l, m, n, p \in \mathbb{N}\right\}, \quad\left\{f_{4^{\prime}}^{l} f_{3^{\prime}}^{m} f_{2^{\prime}}^{n} f_{1^{\prime}}^{p} \mid l, m, n, p \in \mathbb{N}\right\},
$$

where

$$
\begin{aligned}
\left(f_{4}, f_{3}, f_{2}, f_{1}\right) & =\left(F_{\beta}, F_{\beta} F_{\alpha}-v^{2} F_{\alpha} F_{\beta}, \frac{F_{\beta} F_{\alpha}^{2}}{[2]}-v F_{\alpha} F_{\beta} F_{\alpha}+\frac{v^{2} F_{\alpha}^{2} F_{\beta}}{[2]}, F_{\alpha}\right) \\
\left(f_{4^{\prime}}, f_{3^{\prime}}, f_{2^{\prime}}, f_{1^{\prime}}\right) & =\left(F_{\alpha}, \frac{v^{2} F_{\beta} F_{\alpha}^{2}}{[2]}-v F_{\alpha} F_{\beta} F_{\alpha}+\frac{F_{\alpha}^{2} F_{\beta}}{[2]}, F_{\alpha} F_{\beta}-v^{2} F_{\beta} F_{\alpha}, F_{\beta}\right) .
\end{aligned}
$$

It follows from 23 that: 
Lemma A.2 The structure constants of $\mathcal{U}^{-}$, in the basis of $w$, is $q$-holonomic.

Let us define a scalar product $(\cdot, \cdot)$ on $\mathcal{U}^{-}$such that the PBW basis of $w$ is an orthonormal basis. Then Proposition 7.3 is equivalent to

$$
\left(f_{4^{\prime}}^{l^{\prime}} f_{3^{\prime}}^{m^{\prime}} f_{2^{\prime}}^{n^{\prime}} f_{1^{\prime}}^{p^{\prime}}, f_{4}^{l} f_{3}^{m} f_{2}^{n} f_{1}^{n}\right)
$$

is $q$-holonomic in all variables $l, m, n, p, l^{\prime} m^{\prime}, n^{\prime}, p^{\prime}$.

Since multiplication is $q$-holonomic in the PBW basis of $w$ (see Lemma A.2), it suffices to show that

$$
\left(f_{i^{\prime}}^{k}, f_{4}^{l} f_{3}^{m} f_{2}^{n} f_{1}^{p}\right)
$$

is $q$-holonomic in $k, l, m, n, p$ for each $i=1,2,3,4$. This is clear for $i^{\prime}=1$ or $i^{\prime}=4$, since $f_{1^{\prime}}=f_{4}$ and $f_{4^{\prime}}=f_{1}$. As for $i^{\prime}=2$ and $i^{\prime}=3$, the formula of [22, Section 37.1] shows that

$$
\begin{aligned}
& f_{2^{\prime}}^{n}=\sum_{i=0}^{n}(-1)^{i} \frac{v^{2 i} F_{\beta}^{i} F_{\alpha}^{n} F_{\beta}^{n-i}}{[n-i]_{\beta} ![i]_{\beta} !} \\
& f_{3^{\prime}}^{n}=\sum_{i=0}^{2 n}(-1)^{i} \frac{v^{i} F_{\alpha}^{2 n-i} F_{\beta}^{n} F_{\alpha}^{i}}{[2 n-i] ![i] !}
\end{aligned}
$$

and since $F_{\alpha}=f_{4^{\prime}}$ and $F_{\beta}=f_{1^{\prime}}$, the cases of $i^{\prime}=2^{\prime}$ and $i^{\prime}=3^{\prime}$ reduce to the cases of $i^{\prime}=1^{\prime}$ and $i^{\prime}=4^{\prime}$. This proves Proposition 7.3 for $B_{2}$.

Remark A.3 If Lemma A.1 holds for $G_{2}$, then we can prove Proposition 7.3 for $G_{2}$.

\section{References}

[1] D Bar-Natan, ColoredJones.nb, Mathematica program, part of "KnotAtlas", April 2003.

[2] I N Bernšteŭn, Modules over a ring of differential operators. An investigation of the fundamental solutions of equations with constant coefficients, Funkcional. Anal. i Priložen. 5 (1971) 1-16, English translation: 89-101 MathReview

[3] I N Bernšteĭn, Analytic continuation of generalized functions with respect to a parameter, Funkcional. Anal. i Priložen. 6 (1972) 26-40, English translation: 273-285 MathReview

[4] J-E Björk, Rings of differential operators, volume 21 of North-Holland Mathematical Library, North-Holland Publishing Co., Amsterdam (1979) MathReview 
[5] A Borel, P-P Grivel, B Kaup, A Haefliger, B Malgrange, F Ehlers, Algebraic D-modules, Perspectives in Mathematics 2, Academic Press, Boston, MA (1987) MathReview

[6] $\mathbf{P}$ Cartier, Démonstration "automatique" d'identités et fonctions hypergéométriques (d'après D. Zeilberger), Astérisque (1992) Exp. No. 746, 3, 41-91 MathReview

[7] S C Coutinho, A primer of algebraic D-modules, volume 33 of London Mathematical Society Student Texts, Cambridge University Press, Cambridge (1995) MathReview

[8] F Chyzak, B Salvy, Non-commutative elimination in Ore algebras proves multivariate identities, J. Symbolic Comput. 26 (1998) 187-227 MathReview

[9] S Garoufalidis, T T Q Le, D Zeilberger, The quantum MacMahon Master Theorem, arXiv:math.QA/0303319, to appear in Proc. Nat. Acad. Sci.

[10] S Garoufalidis, Difference and differential equations for the colored Jones function, arXiv:math.GT/0306229

[11] S Garoufalidis, On the characteristic and deformation varieties of a knot, from: "Proceedings of the CassonFest (Arkansas and Texas 2003)", Geom. Topol. Monogr. 7 (2004) 291-309

[12] S Garoufalidis, X Sun, The C-polynomial of a knot, preprint (2005) arXiv:math.GT/0504305

[13] R Gelca, Non-commutative trigonometry and the A-polynomial of the trefoil knot, Math. Proc. Cambridge Philos. Soc. 133 (2002) 311-323 MathReview

[14] R Gelca, J Sain, The noncommutative A-ideal of a $(2,2 p+1)$-torus knot determines its Jones polynomial, J. Knot Theory Ramifications 12 (2003) 187201 MathReview

[15] K Habiro, On the quantum $\mathrm{sl}_{2}$ invariants of knots and integral homology spheres, from: "Invariants of knots and 3-manifolds (Kyoto, 2001)", Geom. Topol. Monogr. 4 (2002) 55-68 MathReview

[16] K Habiro, T T Q Le, in preparation

[17] J Jantzen, Lectures on quantum groups, volume 6 of Graduate Studies in Mathematics, American Mathematical Society, Providence, RI (1996) MathReview

[18] L I Korogodski, Y S Soibelman, Algebras of functions on quantum groups. Part I, volume 56 of Mathematical Surveys and Monographs, American Mathematical Society, Providence, RI (1998) MathReview

[19] K Hikami, Difference equation of the colored Jones polynomial for torus knot, Internat. J. Math. 15 (2004) 959-965 MathReview

[20] T T Q Le, Integrality and symmetry of quantum link invariants, Duke Math. J. 102 (2000) 273-306 MathReview

[21] T T Q Le, The Colored Jones Polynomial and the A-Polynomial of Knots, arXiv:math.GT/0407521 
The colored Jones function is $q$-holonomic

[22] G Lusztig, Introduction to quantum groups, volume 110 of Progress in Mathematics, Birkhäuser Boston Inc., Boston, MA (1993) MathReview

[23] G Lusztig, Quantum groups at roots of 1, Geom. Dedicata 35 (1990) 89-113 MathReview

[24] B Malgrange, Équations différentielles à coefficients polynomiaux, volume 96 of Progress in Mathematics, Birkhäuser Boston Inc., Boston, MA (1991) MathReview

[25] G Masbaum, Skein-theoretical derivation of some formulas of Habiro, Algebr. Geom. Topol. 3 (2003) 537-556 MathReview

[26] P Paule, A Riese, A Mathematica q-analogue of Zeilberger's algorithm based on an algebraically motivated approach to q-hypergeometric telescoping, from: "Special functions, $q$-series and related topics (Toronto, ON, 1995)", Fields Inst. Commun. 14, Amer. Math. Soc., Providence, RI (1997) 179-210 MathReview

[27] P Paule, A Riese, Mathematica software, available at: http://www.risc.uni-linz.ac.at/research/combinat/risc/software/qZeil/

[28] M Petkovšek, HS Wilf, Doron Zeilberger, $A=B$, A K Peters Ltd., Wellesley, MA (1996) MathReview

[29] N Yu Reshetikhin, V G Turaev, Ribbon graphs and their invariants derived from quantum groups, Comm. Math. Phys. 127 (1990) 1-26 MathReview

[30] A Riese, qMultisum-A package for proving q-hypergeometric multiple summation identities, preprint (2002)

[31] C Sabbah, Systèmes holonomes d'équations aux q-différences, from: "Dmodules and microlocal geometry (Lisbon, 1990)", de Gruyter, Berlin (1993) 125-147 MathReview

[32] V G Turaev, The Yang-Baxter equation and invariants of links, Invent. Math. 92 (1988) 527-553 MathReview

[33] H S Wilf, D Zeilberger, An algorithmic proof theory for hypergeometric (ordinary and "q") multisum/integral identities, Invent. Math. 108 (1992) 575-633 MathReview

[34] L Yen, A two-line algorithm for proving q-hypergeometric identities, J. Math. Anal. Appl. 213 (1997) 1-14 MathReview

[35] D Zeilberger, A holonomic systems approach to special functions identities, J. Comput. Appl. Math. 32 (1990) 321-368 MathReview 